\title{
MOFs (METAL-ORGANIC FRAMEWORKS): UMA FASCINANTE CLASSE DE MATERIAIS INORGÂNICOS POROSOS
}

\author{
Regina C. G. Frema,*, Guilherme Arroyos ${ }^{a}$, Jader B. da Silva Flor ${ }^{\mathrm{a}}$, Renata C. Alves ${ }^{\mathrm{a}}$, Guilherme N. Lucena ${ }^{\mathrm{a}}$, Caroline M. \\ da Silva ${ }^{a}$ e Mariana F. Coura ${ }^{a}$ \\ aDepartamento de Química Geral e Inorgânica, Instituto de Química, Universidade Estadual de São Paulo, 14800-970 Araraquara \\ - SP, Brasil
}

Recebido em 23/04/2018; aceito em 16/07/2018; publicado na web em 11/09/2018

\begin{abstract}
MOFs (METAL-ORGANIC FRAMEWORKS): A FASCINATING CLASS OF POROUS INORGANIC MATERIALS. Metal-Organic Frameworks (MOFs) are, according to IUPAC, coordination polymers with an open framework containing potential voids, which can be accessible after activation processes. These materials have aroused interest in the academic environment - and more recently in industry - because their properties such as high crystallinity, permanent microporosity, high surface areas, and the possibility of functionalization. This has enabled applications in different areas, such as gas adsorption, catalysis, drug delivery, photonics and others. Depending on the judicious choice of the metals and the organic linkers, MOFs with different topologies and functions can be created. Synthetic routes for the preparation of these materials will be discussed, highlighting those in consonance with the Green Inorganic Chemistry. Strategies for controlling the size and morphology of the crystals will also be presented, as well the most recent methodologies for the large-scale production. This review will also discuss the use of MOFs in the context of energy and environment, particularly referring to the adsorption and/or transformation of $\mathrm{CO}_{2}$, storage of $\mathrm{H}_{2}$ and $\mathrm{CH}_{4}$ as well potable water capture.
\end{abstract}

Keywords: MOFs; porous materials; reticular chemistry; green inorganic synthesis; environment and energy.

\section{INTRODUÇÃO}

As MOFs (Metal-Organic Frameworks) consistem em uma classe de materiais porosos, cujo campo de pesquisa tem crescido muito rapidamente nas últimas duas décadas, particularmente após o trabalho icônico de Omar Yaghi e coautores ${ }^{1}$ sobre um composto de $\mathrm{Zn}$ (II) contendo 1,4-benzeno-dicarboxilato (tereftalato) como ligante, chamado MOF-5. Estes materiais são formados a partir da interação entre íons ou clusters metálicos e ligantes orgânicos multitópicos. Deve-se salientar que a ligação química metal-ligante envolvida na formação de MOFs é de natureza predominantemente covalente do tipo ácido/base de Lewis (íon metálico e ligante, respectivamente), visto que se tratam de compostos de coordenação. ${ }^{2}$

Após 1999, mais do que uma centena de artigos de revisão ${ }^{3-7} \mathrm{e}$ alguns livros ${ }^{8-13}$ têm sido publicados envolvendo o design e a síntese de MOFs, análises estrutural e topológica, propriedades e aplicações, além de estudos de modelagem molecular. Com relação a este último tópico, os principais grupos de pesquisa em MOFs têm usado predominantemente a dinâmica molecular clássica para estudar os tipos de interação com moléculas gasosas, embora alguns estudos utilizando a simulação de estrutura eletrônica baseada na Teoria do Funcional da Densidade (DFT) também estão sendo realizados., ${ }^{3,8}$

Do ponto de vista do ensino de Química, periódicos como o Journal of Chemical Education vêm demonstrando a importância da realização de experimentos sobre o tema MOFs em disciplinas de Química Inorgânica, ${ }^{14,15}$ já estando inclusive na capa de um importante livro-texto de Química Geral. ${ }^{16}$ No que diz respeito à material de referência sobre o assunto disponível em língua portuguesa, do que seja do nosso conhecimento, existem apenas dois artigos publicados na Química Nova ${ }^{17,18}$ e um livro coordenado pelo professor Severino Alves, da Universidade Federal de Pernambuco. ${ }^{19}$ Portanto, a grande motivação para a publicação desse artigo de revisão é colaborar na

*e-mail: rcgfrem@iq.unesp.br disponibilização, para nossos jovens pesquisadores e estudantes brasileiros, de um texto conciso e atualizado, em língua portuguesa, sobre o estado da arte dessa nova classe de materiais inorgânicos porosos, e que possa, dessa forma, despertar o interesse para essa importante e atual área do conhecimento. De fato, o grande interesse nesse tipo de material, que praticamente não é encontrado na natureza - com exceção dos minerais stepanovita e zhemchuzhnikovita ${ }^{20}$ - está relacionado com o conjunto de suas propriedades bem características. Alta cristalinidade, microporosidade permanente (em sua grande maioria), valores elevados de área superficial, baixa densidade, boas estabilidades térmica e química, ${ }^{21,22}$ além da possibilidade de funcionalização de seus poros, tornam as MOFs altamente atrativas em pesquisa de fronteira. No que diz respeito à funcionalização desses materiais, importante destacar a predominância de ligações covalentes nesse processo, que podem ocorrer no ligante e/ou no metal, sendo este último caso apenas possível se houver sítios de coordenação insaturados. Dentro dessa perspectiva, estudos vêm sendo realizados envolvendo aplicações desse tipo de material em diferentes áreas como armazenamento ${ }^{23,24}$ e separação de gases, ${ }^{25}$ catálise heterogênea, ${ }^{26}$ liberação de fármacos,${ }^{27}$ fotônica, ${ }^{28}$ entre outras. ${ }^{29-32}$

A partir de 2012, a Divisão de Química Inorgânica da IUPAC (União Internacional de Química Pura e Aplicada) tem recomendado o uso da definição oficial de MOF como "uma rede de coordenação com ligantes orgânicos contendo cavidades potencialmente vazias"., 2,33 Do ponto de vista histórico, o primeiro trabalho acerca dessa classe de materiais foi publicado por Kinoshita e colaboradores em 1959. ${ }^{34}$ Esse artigo envolveu a síntese de um sólido de coordenação construído a partir de interações covalentes entre íons $\mathrm{Cu}(\mathrm{I})$ tetraédricos e adiponitrila como ligante. Trinta anos mais tarde, Hoskins e Robson publicaram o primeiro artigo no qual foi introduzido o conceito de estruturas poliméricas porosas baseadas em ligações covalentes metal-ligante. ${ }^{35}$ Usando o modelo nod-and-spacer proposto por Wells em 1978, ${ }^{36}$ um sólido de coordenação 3D foi obtido, consistindo de centros tetraédricos ligados por unidades de construção lineares. Dez 
anos se passaram antes que pudesse ter sido evidenciada a prova da porosidade permanente para MOFs. ${ }^{37}$ Esse mesmo grupo de pesquisa, liderado pelo professor Omar Yaghi, preparou o composto de coordenação $\left[\mathrm{Zn}_{4} \mathrm{O}(\mathrm{BDC})_{3}\right]$. $(\mathrm{DMF})_{8}\left(\mathrm{C}_{6} \mathrm{H}_{5} \mathrm{Cl}\right)$, onde $\mathrm{BDC}=1$, 4-benzenodicarboxilato e DMF $=\mathrm{N}, \mathrm{N}^{\prime}$-dimetilformamida, conhecido $^{1}$ como MOF-5. A partir de então, as MOFs começam a se tornar conhecidas no meio acadêmico e observa-se um crescimento vertiginoso dessa área de pesquisa. No que se refere à nomenclatura das MOFs, é bastante comum que elas sejam "batizadas" com uma sigla que representa o nome do lugar onde foram originalmente obtidas, seguido por um número inteiro (n) atribuído em ordem cronológica da descoberta. Como exemplos, podemos citar as MOFs conhecidas como MIL-n (MIL = Matériaux de I'Institut Lavoisier) ${ }^{38}$ HKUST-n $\left(\right.$ HKUST $=$ Hong-Kong University of Science and Technology ${ }^{39}$ ou UiO-n $(\mathrm{UiO}=$ Universitetet $i$ Oslo $){ }^{40}$

\section{Síntese Reticular e Topologias de MOFs}

Visando analisar os fatores responsáveis pela montagem das MOFs a partir de blocos de construção moleculares e que contribuíssem então para o planejamento racional dessa classe de materiais, o grupo do professor Omar Yaghi desenvolveu uma metodologia, baseada nos conceitos da Química Modular, ${ }^{41}$ conhecida como Síntese Reticular. Na estratégia proposta, moléculas orgânicas politópicas se ligam a íons de metais de transição. ${ }^{42}$ Em seguida, os autores relataram o próximo estágio no desenvolvimento das MOFs, introduzindo o conceito de unidades de construção secundárias (secondary building units, $S B U s)^{43}$ que são ligadas por ligações covalentes fortes através de todo o cristal. SBUs podem então ser definidas como "complexos e clusters moleculares nos quais os modos de coordenação do ligante e as geometrias de coordenação do metal podem ser utilizados na transformação desses fragmentos em redes estendidas porosas usando ligantes politópicos". Em 2003, o grupo publica, então, um artigo definitivo no qual fica estabelecida a estratégia conceitual (Síntese Reticular) para a construção racional de MOFs a partir de unidades de construção moleculares. ${ }^{44}$ Química Reticular se refere, portanto, à organização de estruturas ordenadas pré-determinadas através de blocos de construção moleculares rígidos ( $S B U S$ ) que se repetem e se mantém unidos através de ligações metal-ligante. Nesse trabalho, os autores redefinem o termo $S B U$ (originalmente usado para descrever fragmentos de zeólitas), se referindo à geometria das unidades definidas pelos pontos de extensão. Na Figura 1, por exemplo, algumas SBUs são mostradas, onde os pontos de extensão correspondem aos átomos de carbono (em preto) em MOFs baseadas em carboxilatos. ${ }^{45}$ No lado direito dessa mesma figura, pode ser vista uma representação química dos mesmos blocos de construção.

No entanto, para a formação de uma rede estendida, é imprescindível que as unidades de construção secundárias (as $S B U s$ ) sejam posicionadas corretamente na estrutura do material (para garantir a tridimensionalidade) e para isso, a geometria do ligante orgânico passa a desempenhar um papel da maior relevância. Como mostra a Figura 2A, materiais construídos a partir do mesmo bloco de construção quadrado (ver representação a da Figura 1) podem apresentar diferentes dimensionalidades, dependendo da forma do ligante orgânico que os interconecta. Poliedros discretos (uso, nesse caso, do ligante angular 1,3-benzenodicarboxilato) até MOFs 3D, passando por cadeias $1 \mathrm{D}$ e folhas $2 \mathrm{D}$ podem ser formados. ${ }^{46} \mathrm{Na}$ Figura $2 \mathrm{~B}$, está ilustrada a estrutura cristalina da MOF-101 de topologia nbo, construída a partir da combinação entre blocos de construção quadrados do tipo $\mathrm{Cu}_{2}(\mathrm{COO})_{4}$ e o ligante 2-bromo-1,4-benzenodicarboxilato. ${ }^{47}$

Logo, do ponto de vista da química reticular, para uma geometria específica de unidade de construção, MOFs racionalmente projetadas podem ser sintetizadas. Pelo fato de os vértices topologicamente a)
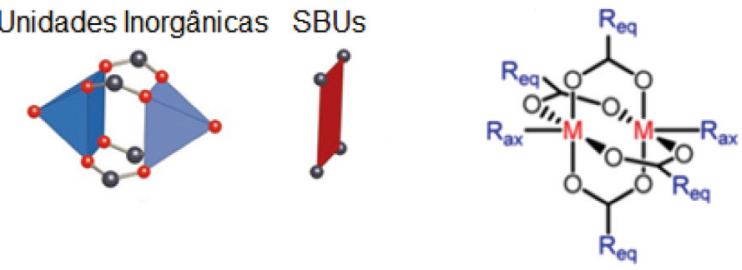

b)
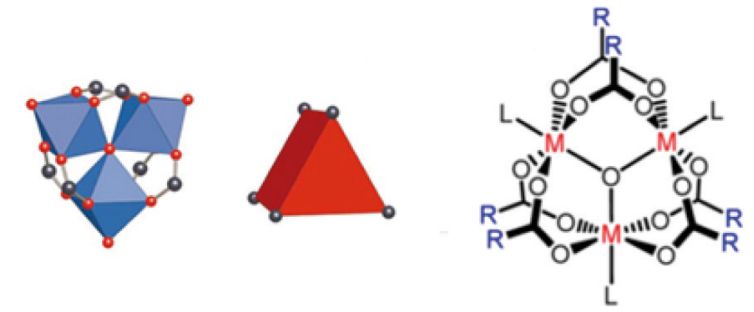

c)
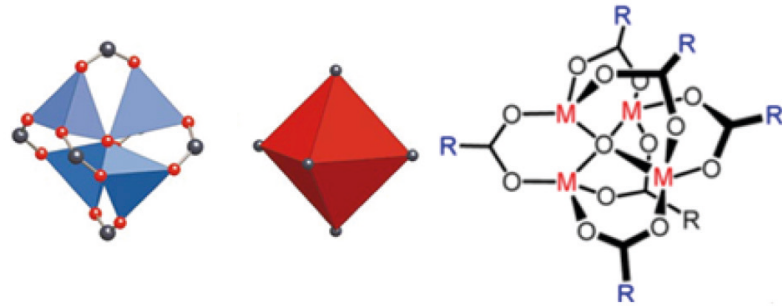

Figura 1. Duas formas diferentes de representar algumas unidades de construção (SBUs, Secondary Building units) de MOFs baseadas em ligantes carboxilatos. À esquerda: $O$, vermelho; $C$, preto; poliedros metal-oxigênio em azul; polígono ou poliedro definidos pelos átomos de carbono do carboxilato (SBUs) em vermelho. À direita: representação química. (a) SBU dinuclear quadrada (paddle-wheel) do tipo $\left[\mathrm{M}_{2}\left(\mathrm{O}_{2} \mathrm{CR}\right)_{4} L_{2}\right](\mathrm{M}=$ metal de transição, $L=$ ligante axial) com quatro grupos carboxilatos em ponte, (b) $S B U$ trimetálica $\mu$ 3-oxo do tipo $\left[\mathrm{M}_{3} \mathrm{O}\left(\mathrm{O}_{2} \mathrm{CR}\right)_{6} \mathrm{~L}_{3}\right]$ com seis carboxilatos em ponte, (c) SBU tetrametálica $\mu$ 4-oxo $\left[\mathrm{M}_{4} \mathrm{O}\left(\mathrm{O}_{2} \mathrm{CR}\right)_{6}\right]$, protótipo de um octaedro molecular. Reproduzido das referências 44 e 45 com permissão da Nature e American Chemical Society

diferentes e a conectividade entre eles serem praticamente infinitos, o número de estruturas possíveis seria, em princípio, ilimitado. Contudo, o ponto central desse modelo é que, de acordo com os princípios do design geométrico, há um número relativamente pequeno de topologias básicas (default nets) que podem ser formadas. Em outras palavras, dependendo da geometria e conectividade dos blocos de construção, as MOFs sintetizadas apresentarão topologias básicas simples. ${ }^{48,49} \mathrm{E}$ a partir dos dados topológicos coletados, as redes mais comuns podem ser identificadas através da base de dados Reticular Chemistry Structure Resource (RCSR)..$^{50}$ No momento em que esse artigo está sendo redigido (abril de 2018) mais do que 2800 redes $3 \mathrm{D}$ diferentes estão descritas na base RCSR. ${ }^{50}$ No entanto, a maioria delas apresentam topologias básicas muito simples para uma determinada forma e conectividade dos blocos de construção. A título de ilustração, a Tabela 1 lista topologias comuns (default nets) com um ou dois tipos de $S B U$ s e MOFs representativas de cada uma delas. ${ }^{48,49}$ Deve ser destacado que os códigos de três letras que identificam as redes que descrevem as topologias das MOFs podem ser abreviações de um tipo de estrutura $(\mathbf{p c u}=$ primitive cubic $)$, nome de um mineral (dia = diamante) ou a forma contraída da fórmula de compostos químicos, como srs que corresponde à rede de átomos de silício em $\mathrm{SrSi}_{2} \cdot{ }^{51-53}$

Importante informar que, na mesma época em que Yaghi e O'Keeffe desenvolviam os primeiros trabalhos sobre MOFs (início

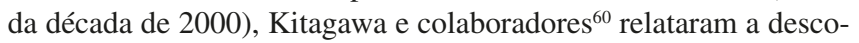
berta do sólido $3 \mathrm{D}$ altamente poroso $\left[\left\{\mathrm{CuSiF}_{6} 4,4^{\prime} \text {-bipiridina }{ }_{2}\right\}_{\mathrm{n}}\right]$, 
a)
OD

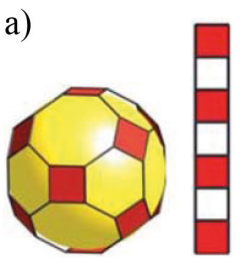

$1 D$

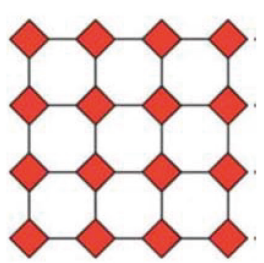

2D

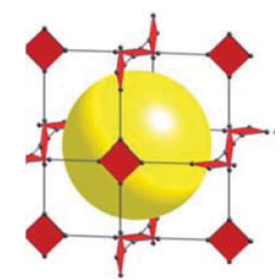

3D b)

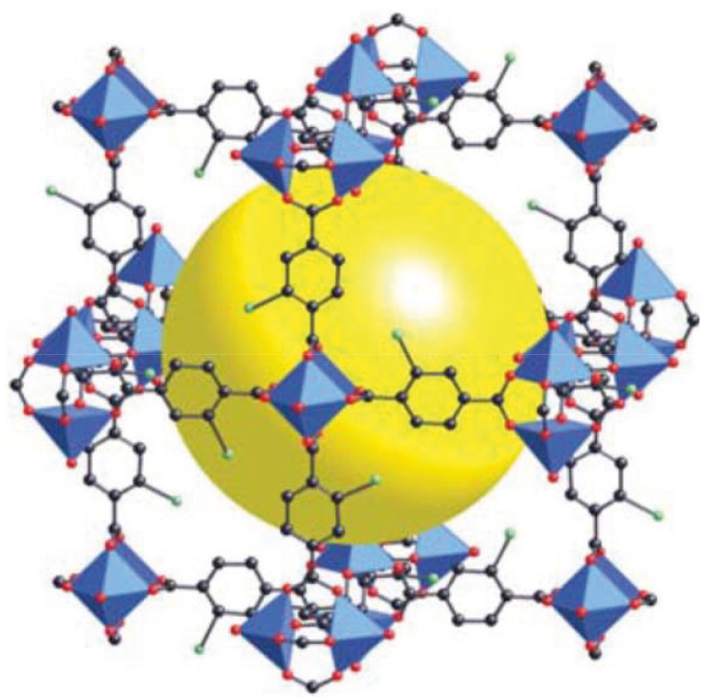

Figura 2. (a) Nanopartícula, cadeia, folha e $3 D$ rede estendida formadas pelas interconexões entre a mesma SBU quadrada do tipo paddlewheel (vermelho) e ligantes ditópicos de diferentes geometrias (preto), (b) Estrutura cristalina do $\left[\mathrm{Cu}_{2}\left\{\mathrm{o}-\mathrm{Br}-\mathrm{C}_{6} \mathrm{H}_{3}\left(\mathrm{CO}_{2}\right)_{2}\right\}_{2} 2 \mathrm{H}_{2} \mathrm{O}\right] .(\mathrm{DMF})_{8} .\left(\mathrm{H}_{2} \mathrm{O}\right)_{2}(\mathrm{MOF}-101) \mathrm{com}$ topologia nbo. $\mathrm{Cu}$, azul; $\mathrm{O}$, vermelho; $\mathrm{C}$, preto; $\mathrm{Br}$, verde. A esfera amarela representa uma das cavidades na estrutura do material. Reproduzido das referências 46 e 47 com permissão da Cambridge University Press and American Chemical Society

construído através de uma estratégia sintética análoga baseada em blocos de construção.

De qualquer modo, convém destacar aqui que o modelo da química reticular só é capaz de prever a estrutura e a topologia da rede de uma MOF se seus blocos de construção tiverem uma forte tendência em formar clusters específicos tais como $\left[\mathrm{Zn}_{4} \mathrm{O}(\mathrm{COO})_{6}\right]$ na MOF-5, por exemplo. Reações envolvendo os mesmos íons metálicos e ligantes podem de outro modo levar à formação de MOFs completamente diferentes, dependendo da rota sintética e/ou das condições de reação usadas para obter o material. Também é muito difícil projetar a síntese de uma MOF cuja estrutura consiste de $S B U$ s de formas não bem definidas, por exemplo. Dentro desse contexto, Yaghi e O'Keeffe publicaram em 2012 um trabalho de revisão onde mostram como reduzir estruturas de alta complexidade às topologias básicas. ${ }^{61}$

MOFs estruturalmente mais complexas podem ser desenhadas e construídas usando a estratégia conceitual conhecida como $S B B$ (supermolecular building block) proposta por Zaworotko. ${ }^{62-64}$ Essa concepção de engenharia de cristal é baseada no uso de poliedros metal-orgânicos (MOPs) grandes e de alta simetria como blocos de construção (SBBS) para a construção de uma nova classe de MOFs com alto nível de controle sobre a topologia e um novo nível de escala (vários nanômetros) para a estrutura resultante. Apenas para dar um exemplo, a Figura 3 ilustra uma MOF porosa com topologia pcu, construída de poliedros discretos do tipo $\left[\mathrm{Cu}_{6} \mathrm{~L}_{8}\right]\left(\mathrm{NO}_{3}\right)_{12}(\mathrm{~L}=$ N,N',N"-tris3-piridinil--1,3,5-benzenotricarbóxiamida) como unidades de construção $(S B B s) .{ }^{65}$ Este composto orgânico (L) de simetria- $\mathrm{C}_{3}$ atua como ligante facial triangular enquanto íons $\mathrm{Cu}(\mathrm{II})$ tetragonalmente distorcidos podem ser considerados os vértices do octaedro molecular. Os contraíons nitrato ligam esses vértices metálicos, uma vez que o sítio axial do íon $\mathrm{Cu}$ (II) está direcionado para o exterior dos nanocages, servindo como um nó de conexão para a formação da rede 3D. Como também pode ser observado na Figura 3, dois tipos diferentes de nanocages (representados pelos octaedros amarelos e verdes) interagem entre si através de forças intermoleculares do tipo $\pi . . . \pi$ stacking entre os anéis aromáticos centrais dos ligantes. No entanto, a MOF retém as cavidades em escala nanométrica, que originalmente pertenciam aos poliedros de construção (MOPs). Mais recentemente, Eddaoudi e Lah introduziram outra estratégia de design para a construção de MOFs de alta complexidade estrutural baseada no conceito de supermolecular building layer (SBL) ${ }^{66,67}$ A partir dessa metodologia, é possível obter redes tridimensionais de diversas topologias via o empilhamento de MOFs bidimensionais usando, por exemplo, os sítios metálicos axiais disponíveis na vizinhança e ligantes ditópicos.

Há uma classificação recente das $\mathrm{MOFs}^{68}$ que leva em conta o efeito exercido pela molécula ou íon-hóspede em seu interior e está representada na Figura 4.

De fato, as MOFs relatadas à época do trabalho pioneiro de Yaghi e O’Keeffe 1999. possuíam apenas um íon metálico como vértice da rede metalorgânica. Estes íons não eram robustos o suficiente para manter a rigidez estrutural quando se removiam as moléculas de solvente oclusas nos poros. Portanto, ao dessorver estas moléculas, a estrutura colapsava, sendo então possível utilizá-la apenas uma única vez. ${ }^{69}$ Estas MOFs foram classificadas como de primeira geração e estão representadas no canto superior esquerdo da Figura 4. Por outro lado, quando os vértices da estrutura são compostos pelas unidades de construção secundária (secondary building units, $S B U s),{ }^{44}$ na realidade clusters metálicos rígidos, formam-se MOFs categorizadas como de segunda geração, ${ }^{1}$ também ilustradas na Figura 4. Estas MOFs possuem, então, estrutura rígida, além de porosidade permanente, ao se remover moléculas de solvente adsorvidas em suas cavidades. No que diz respeito às MOFs de terceira geração, estas apresentam uma certa flexibilidade estrutural como principal característica. ${ }^{70}$ Esta flexibilidade faz com que a estrutura se deforme reversivelmente quando uma substância é adsorvida ou por efeito de um estímulo externo (luz, temperatura ou campo elétrico). Estes materiais são chamados de breathing MOFs ou MOFs dinâmicas. ${ }^{71}$ As MOFs de quarta geração são aquelas que podem sofrer modificações pós-sintéticas sem alterar a topologia do material. Estas funcionalizações consistem em introduzir grupos funcionais (moléculas ou íons) na estrutura da MOF já sintetizada visando reconhecimento molecular de sítios específicos. ${ }^{72-74}$ Ao contrário do processo de adsorção de hóspedes nos poros, na modificação pós-sintética, a substância introduzida passa a fazer parte da estrutura do material. As MOFs de terceira e quarta gerações estão representadas na parte inferior da Figura 4.

\section{ESTRATÉGIAS DE PREPARAÇÃO DE MOFS EM PEQUENA E GRANDE ESCALA}

Várias rotas sintéticas para a preparação de MOFs têm sido apresentadas e discutidas na literatura. ${ }^{75-78}$ A maioria consiste de métodos em solução tais como mistura direta de íons metálicos solúveis e ligantes orgânicos (via adição direta ou difusão lenta), ${ }^{79}$ solvotérmico (usando aquecimento elétrico), ${ }^{80}$ síntese assistida por microondas (também sob condições solvotérmicas), ${ }^{81}$ sonoquímico ${ }^{82}$ e eletroquímico ${ }^{83,84}$ A síntese solvotérmica, por exemplo, embora muito importante para a obtenção e crescimento de monocristais adequados para resolução estrutural por difratometria de raios-X (DRX), necessita de tempos prolongados de reação (alguns dias) e 
Tabela 1. Algumas topologias comuns e MOFs representativos relacionados ( $\mathrm{NC}=$ número de coordenação)

\begin{tabular}{|c|c|c|c|c|c|c|}
\hline $\mathrm{NC}$ vértices & Form & SBUs & Rede (código) & Exemplo & Ref. & Representação \\
\hline 3 & Triângulo & Triângulo & $\mathrm{SrSi}_{2}(\mathbf{s r s})$ & {$[\mathrm{CuL}(\mathrm{DMF})(\mathrm{py})]^{\mathrm{a}}$} & 54 & \\
\hline 3 & Triângulo & Triângulo & $\mathrm{ThSi}_{2}$ (ths) & {$\left[\left(\mathrm{ZnI}_{2}\right)_{3}(\mathrm{TPT})_{2}\right]^{\mathrm{b}}$} & 55 & \\
\hline 3 & Triângulo & Triângulo & Honeycomb (hcb) & CPO-27-M & 56 & \\
\hline 3,4 & Triângulo & quadrado & twisted boracite (tbo) & HKUST-1 & 39 & \\
\hline 3,4 & Triângulo & quadrado & $\mathrm{Pt}_{3} \mathrm{O}_{4}(\mathbf{p t o})$ & MOF-388 & 57 & \\
\hline 4 & Quadrado & quadrado & square lattice (sql) & MOF-118 & 58 & \\
\hline 4 & Tetraedro & Tetraedro & diamond (dia) & {$\left[\mathrm{CuC}\left(\mathrm{C}_{6} \mathrm{H}_{4} \cdot \mathrm{CN}\right)_{4}\right]^{+}$} & 35 & \\
\hline 4,4 & Quadrado & Tetraedro & PtS (pts) & {$\left[\mathrm{Cu}_{2} \mathbf{L}^{\prime}\left(\mathbf{H}_{2} \mathbf{O}\right)_{2}\right]^{\mathrm{c}}$} & 59 & \\
\hline 6 & Octaedro & Octaedro & primitive cubic (pcu) & MOF-5 & 44 & \\
\hline
\end{tabular}

${ }^{\mathrm{a}} \mathrm{L}=3,5$ - piridinadicarboxilato; $\mathrm{py}=$ piridina. ${ }^{\mathrm{b}} \mathrm{TPT}=2,4,6$-tris $\left(4\right.$-piridil)triazina. ${ }^{\mathrm{c}} \mathrm{L}$ ' $=$ tetrakis[4-(carbóxifenil)oximetil]metano.

temperaturas relativamente altas $(\sim 400 \mathrm{~K})$, além do uso de solventes caros, tóxicos e de alto ponto de ebulição como a dimetilformamida. Além disso, a formação de fases impuras (moléculas do ligante em excesso ou recristalizadas, por exemplo) resulta em rendimentos baixos quando a MOF é preparada por essa técnica. De qualquer forma, do ponto de vista ambiental, sínteses de estado sólido são muito mais adequadas e dentro desse contexto, se destaca a síntese mecanoquímica $^{85,86}$ (em almofariz ou reator específico) que ocorre a temperatura ambiente, é muito rápida (minutos) e virtualmente livre de solvente. ${ }^{87}$ Mais recentemente, para evitar o uso de compostos metálicos solúveis (e a formação de subprodutos acídicos), tem sido proposto o uso de precursores metálicos sólidos como hidróxidos ou óxidos, e assim é possível preparar MOFs tendo apenas água como o único subproduto. ${ }^{88}$ Sob essa mesma perspectiva, a partir de 2011, a conversão de gel seco vem sendo investigada ${ }^{89-91}$ para obter MOFs em alto rendimento, usando um volume de reação bastante reduzido e gerando quantidade mínima de rejeitos.

Produção em grande escala. Para produzir grandes quantidades de MOFs para o mercado, algumas rotas sintéticas têm sido investigadas. Os métodos para manufatura em grande escala de produtos baseados em MOFs precisam preencher certos requisitos 
a)

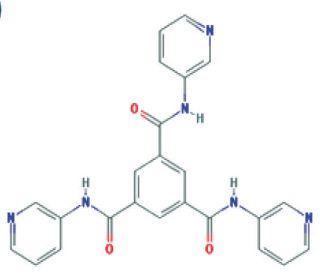

c)

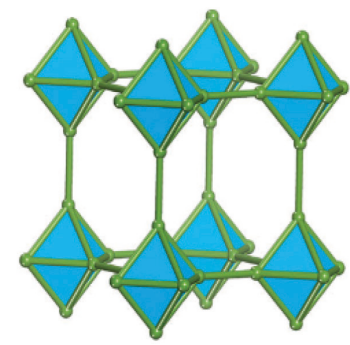

b)

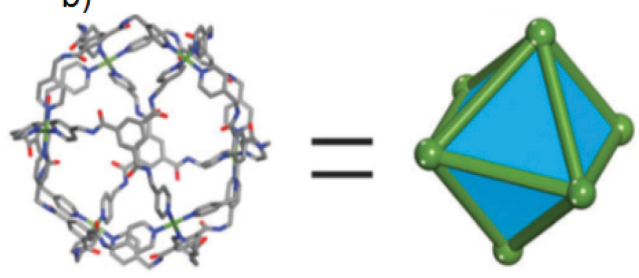

d)

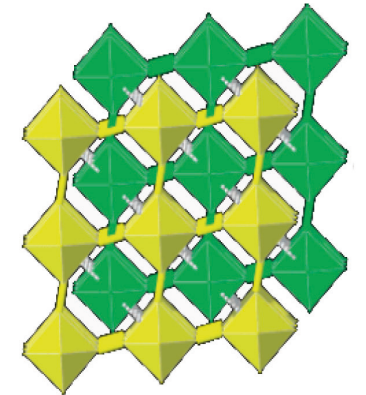

Figura 3. (a) ligante $N, N^{\prime}, N$ "-tris(3-piridinil)-1,3,5-benzenotricarbóxiamida; (b) octaedro discreto $(M O P)$ do tipo $\left[\mathrm{Cu}_{6} L_{8}\right]^{12+}$ com faces (em azul) representando os C3-ligantes e vértices (em verde) representando os íons $\mathrm{Cu}^{2+}$; (c) uma rede cúbica primitive (pcu) obtida pela ligação entre as MOPs e ânions nitrato; (d) diagrama de empacotamento esquemático da estrutura cristalina, mostrando o nitrato ditópico como bastões verdes ou amarelos e a interação intermolecular do tipo $\pi$... $\pi$, como pontos em cinza. Reproduzido das referências 63 e 65 com permissão da The Royal Society of Chemistry e American Chemical Society

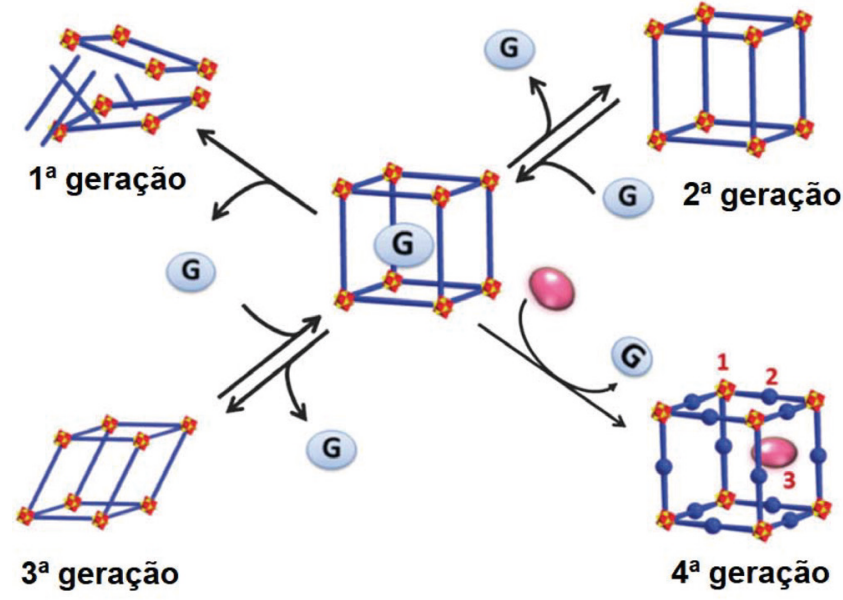

Figura 4. Representações estruturais do efeito da remoção de uma molécula ou íon hóspede $(G)$ dos poros de MOFs de diferentes gerações. Legenda: aresta azul = ligantes; poliedro vermelho $=$ SBU metálica; esfera azul $=$ modificação pós-sintética; elipse rosa: espécie de interesse. Adaptada da referência 68 com permissão da The Royal Society of Chemistry

como reprodutibilidade, eficiência (rendimentos altos), economia (matéria-prima barata) e compromisso ambiental (alta pureza do produto e baixo consumo de energia). A partir de 2005, quando foi relatado pela primeira vez o uso da rota eletroquímica para preparar HKUST-1, ${ }^{22}$ a BASF vem produzindo essa MOF de cobre em escala de tonelada (Basolite C300, comercializada pela Sigma-Aldrich) ${ }^{78}$ usando esse método que é versátil e de baixo custo. MOFs também podem ser produzidas de modo contínuo usando reatores de fluxo, ${ }^{93,94}$ método solvotérmico, ${ }^{95,96}$ técnicas de fluxo contínuo como aerosol ou spray-drying (ver Figura 5) ) $^{97,98}$ e rota mecanoquímica. A partir dessa metodologia de estado sólido, tem sido possível a síntese por extrusão para a produção em larga escala de alguns tipos de MOFs. ${ }^{99}$

Há também alguns trabalhos interessantes sobre processos de produção de MOFs por batelada. ${ }^{100-103}$ Contudo, independente do processo de produção, as MOFs são obtidas invariavelmente como pós insolúveis e diversas técnicas (extrusão, uso de aglutinantes, a)
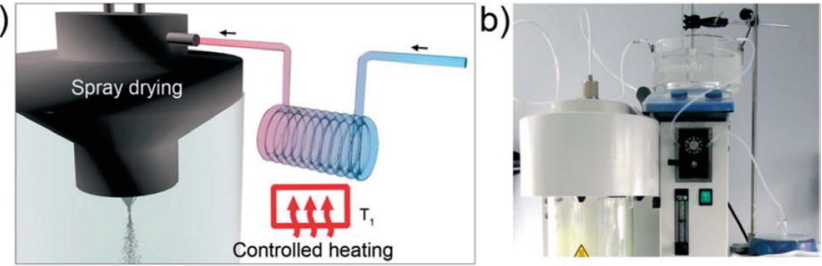

Figura 5. (a) Ilustração esquemática e (b) fotografia mostrando o equipamento para a síntese em fluxo contínuo por spray-drying da MOF UiO-66. Reproduzido da referência 97 com permissão da The Royal Society of Chemistry

pressão mecânica, etc.) têm possibilitado processá-los em formas macroscópicas específicas, tais como extrudatos, pellets, pérolas, monolitos, ${ }^{97,104-106}$ entre outras arquiteturas até mais complexas. ${ }^{107,108}$ Logo, controlando espacialmente o processo de nucleação em posições determinadas, é possível estruturar MOFs em superestruturas ordenadas (core shell, por exemplo), o que abre certamente uma nova oportunidade para melhorar o desempenho do material. Sob essa mesma perspectiva e visando aplicações industriais, ${ }^{109,110}$ MOFs têm também sido empregadas na produção de filmes finos cristalinos, ${ }^{111-113}$ membranas, ${ }^{114,115}$ e compósitos. ${ }^{116,117}$

\section{NUCLEAÇÃO E CRESCIMENTO DE MOFs}

Em princípio, as MOFs podem ser sintetizadas de maneira a produzir cristais com estrutura, forma e tamanho desejados. Avanços nas áreas de cristalografia e engenharia de cristais permitiram a obtenção de partículas com morfologia ou tamanho adequados para melhorar o desempenho das MOFs em diversas aplicações, além de facilitar a sua implementação em dispositivos funcionais. ${ }^{78} \mathrm{~A}$ formação de um cristal de MOF depende diretamente da natureza dos ligantes, metais e solventes usados na síntese, das concentrações dos reagentes e de parâmetros experimentais (temperatura, $\mathrm{pH}$, tempo de reação, etc.), além da rota sintética utilizada. A morfologia da partícula formada (hábito cristalino) nem sempre reflete sua estrutura de retículo. Como exemplo, podemos citar a MOF de $\mathrm{Cu}$ (II) HKUST-1 que possui estrutura cúbica de face centrada mas pode cristalizar na forma de partículas octaédricas. ${ }^{118,119}$ Existem na literatura diversos artigos 
tratando dos processos de cristalização de MOFs. ${ }^{76,120,121}$ Gascon e colaboradores, por exemplo, propuseram que a cristalinidade do produto está diretamente ligada a concentração dos reagentes. A solução inicial deve-se encontrar com uma supersaturação mínima dos reagentes para que ocorra a nucleação. Durante o processo de nucleação podem ser formados núcleos cristalinos ou amorfos, que por sua vez crescerão e formarão os cristais de MOFs. Este crescimento pode ocorrer via três mecanismos, relacionados à supersaturação da mistura reacional de partida. O crescimento espiral (spiral growth) ocorre em condições de baixas supersaturação e concentrações dos reagentes. O crescimento "birth and spread growth" ocorre com supersaturação um pouco maior; neste caso, formam-se vários núcleos sobre a superfície da partícula formada, sendo que estes núcleos propagam-se formando as faces do cristal. Por fim, o crescimento adesivo (adhesive growth) ocorre em alta supersaturação com incorporação de núcleos na superfície da partícula formada, que por sua vez crescem em diversas direções e orientações cristalográficas. Dependendo destes processos podem ser formadas partículas com faces lisas ou rugosas, dependendo também da velocidade de crescimento do material, como pode ser visualizado na Figura 6.

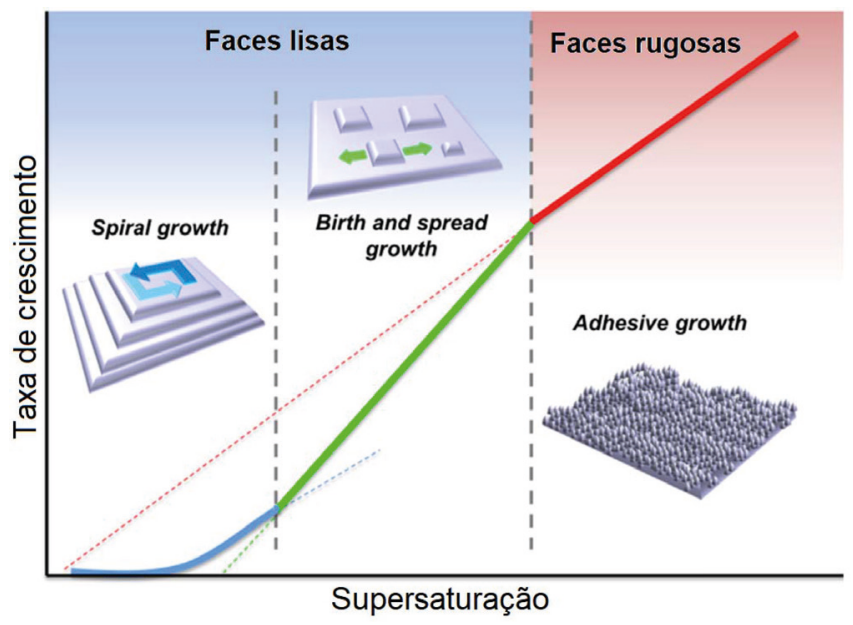

Figura 6. Possíveis tipos de crescimento de cristais de MOFs, dependendo do grau de supersaturação da mistura reacional. Adaptada da referencia 76 e reproduzida com permissão da Elsevier

Em geral, as MOFs apresentam alta cristalinidade, no entanto estes processos de crescimento das partículas podem afetar diretamente a cristalinidade do composto formado. Quando ocorre crescimento adesivo, em condição de alta supersaturação, podem ser formados polímeros de coordenação infinitos (ICP) que se organizam na forma de partículas esféricas amorfas. ${ }^{122}$

\section{Controlando o tamanho e a forma das partículas}

Como esperado para a maioria dos nanomateriais, MOFs obtidas em escala nanométrica exibem propriedades físicas diferentes das dos materiais de origem (sólido bulk). ${ }^{123,124}$ Por essa razão, dependendo da aplicação que se pretenda, é mais apropriado que as MOFs sejam miniaturizadas em escala nanométrica. ${ }^{125} \mathrm{Da}$ mesma forma, é também importante conhecer métodos de controle sobre a morfologia de nano- e microcristais de MOFs. ${ }^{126}$ A Figura 7 mostra uma imagem de microscopia eletrônica de transmissão de alta resolução de um nanocristal monodisperso do material Zn-MOF-74, ${ }^{127}$ onde os clusters metálicos e os canais hexagonais de diâmetro $\sim 11$ Å podem ser observados. De acordo com os autores, essa foi a primeira vez que dados de microscopia eletrônica permitiram a observação de um cluster metálico ligando a fase orgânica em uma MOF.
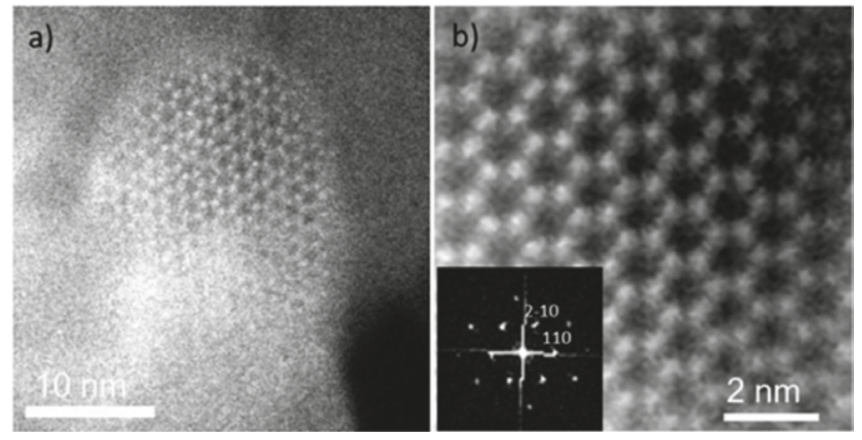

Figura 7. (a) Imagem de Microscopia Eletrônica de Transmissão de altíssima resolução de uma nanopartícula $(20 \mathrm{~nm})$ de $\mathbf{Z n - M O F - 7 4 ~ o r i e n t a d a ~ a o ~ l o n g o ~}$ da direção [001], mostrando um arranjo hexagonal dos poros; (b) uma visão mais aproximada dos poros da partícula, onde os clusters de Zn podem ser visualizados. Reproduzido da referência 127 com permissão da American Chemical Society

Embora essa MOF tenha sido obtida através da mistura direta dos materiais de partida e em princípio, os métodos usados para sintetizar MOFs em escala micrométrica ${ }^{128}$ possam ser usados para preparar nanoMOFs, há alguns métodos específicos para a síntese de partículas de MOFs com tamanho e forma controlados. ${ }^{129,130} \mathrm{Um}$ deles usa moduladores de coordenação ${ }^{131,132}$ onde ligantes monodentados são introduzidos no meio reacional para competir diretamente com as moléculas do linker (polidentado) pelos sítios de coordenação nos blocos de construção. Ácidos monocarboxílicos, como o ácido acético ou fórmico, podem ser usados como moduladores, desempenhando importante papel no controle da nucleação e crescimento das partículas. Kitagawa e co-autores publicaram um estudo interessante no início dessa década ${ }^{133}$ no qual foi demonstrada a transição morfológica de cristais de HKUST-1 (de octaedros para nanopartículas cúbicas) com o aumento na concentração do modulador (ácido $n$-dodecanóico ou láurico nesse caso). Como alternativa, um grande excesso do linker pode também ser usado para esse mesmo propósito. ${ }^{134}$ Em qualquer caso, ambos os mecanismos de modulação aumentam a reprodutibilidade da síntese e a cristalinidade das nanopartículas obtidas. Outra possibilidade para modular o tamanho e a morfologia de partículas de MOFs envolve a preparação de micelas reversas através da mistura de dois líquidos imiscíveis na presença de um surfactante. Nesse caso, as microemulsões obtidas atuam como nanoreatores químicos confinando a síntese da MOF a escala nanométrica. ${ }^{135}$ Além disso, ajustando a concentração do surfactante, Sun e colaboradores demonstraram que é também possível controlar a morfologia da nanopartícula e desse modo, cubos, cubos truncados, cuboctaedros e octaedros puderam ser obtidos. ${ }^{136}$ No entanto, deve ser destacado que o uso do método de microemulsão fornece rendimentos baixos e pouca reprodutibilidade, provavelmente pelo grande número de parâmetros envolvidos no processo de formação da micela. Para evitar a necessidade de adição de solventes imiscíveis ou surfactantes, Maspoch e colaboradores ${ }^{137}$ propuseram o uso da técnica de secagem por aspersão (spray-drying) para a preparação de microcápsulas. O método consiste na dispersão de gotículas contendo os precursores em uma superfície aquecida podendo, dessa forma, ser usado como uma forma de encapsulamento.

\section{Controlando o tamanho e a funcionalidade dos poros}

Apesar da vasta literatura sobre essa excitante área do conhecimento, ainda são raros trabalhos em língua portuguesa acerca de dois aspectos muito importantes quando nos referimos a aplicações de MOFs: o tamanho e a superfície da parede do poro. De fato, para algumas aplicações, especialmente armazenagem/separação de gases 
e catálise heterogênea, os microporos muito pequenos da grande maioria das MOFs podem ser um problema no transporte de massa, por exemplo. Por essa razão, algumas estratégias têm sido usadas para introduzir grandes poros $(>2 \mathrm{~nm})^{138,139}$ objetivando a construção de MOFs mesoporosas ${ }^{140-142}$ e também materiais macroporosos ${ }^{143}$ tais como cápsulas, microesferas ocas, etc. O uso de linkers mais longos ${ }^{144,145}$ foi um dos primeiros métodos usados para aumentar o tamanho dos poros de uma MOF, embora em alguns casos isso tenha levado à formação de estruturas interpenetradas (catenação), ${ }^{146-148}$ reduzindo a área superficial disponível, o tamanho de poro e a estabilidade estrutural. $\mathrm{O}$ uso de surfactantes como agentes direcionadores de estrutura (template) pode ser uma alternativa, e nesse caso em geral mesoporos estarão presentes, além dos microporos. ${ }^{149-151}$

Além da possibilidade de modular o tamanho do poro de uma MOF, é também possível funcionalizar a superfície do poro, ligando grupos funcionais aos linkers orgânicos e, dessa forma, conferir novos níveis de funcionalidade ao material. ${ }^{152}$ Dentro desse contexto, Yaghi e co-autores ${ }^{153}$ propuseram um método que permite prever a performance de uma mesma MOF, para adsorção de um gás, por exemplo, após a incorporação de diferentes combinações de grupos químicos funcionais. Várias funcionalidades diferentes também podem ser introduzidas em uma MOF, com retenção total de sua cristalinidade e porosidade, através de um processo conhecido como modificação pós-sintética (PSM), isto é, a modificação química é realizada após a MOF ter sido sintetizada. ${ }^{73,154,155}$ A funcionalização da parede do poro pode ocorrer por uma interação covalente entre grupos funcionais e os linkers orgânicos e/ou através dos sítios metálicos coordenativamente insaturados dentro da estrutura da MOF, entre outras estratégias. ${ }^{156-159}$
Além da química covalente dentro dos poros, por assim dizer, novas propriedades tais como acidez/basicidade ou magnetismo podem ser introduzidas pela encapsulação de nanopartículas inorgânicas nos poros das MOFs. ${ }^{160-162}$ Essa estratégia sintética para modificar a arquitetura dos poros tem conferido novos desempenhos às MOFs, principalmente na área de catálise heterogênea. ${ }^{163}$

\section{MOFs, ENERGIA E MEIO-AMBIENTE}

As propriedades físicas e químicas características das MOFs tornam-nas materiais relevantes para uma gama de aplicações. ${ }^{164-166}$ Importantes e atuais trabalhos de referencia nas áreas de biomedicina, ${ }^{167-170}$ fotônica, ${ }^{28,171-173}$ adsorção ${ }^{174,175}$ e separação de gases, ${ }^{176,177}$ catálise, ${ }^{26,178-180}$ entre outras, ${ }^{181-183}$ podem ser facilmente acessados. No entanto, considerando a relevância da descoberta de novos materiais que possam atuar na área de Energia e Meio Ambiente, a última parte desse artigo de revisão será devotada ao uso de algumas MOFs no armazenamento de hidrogênio, metano e água, assim como na captura seletiva de $\mathrm{CO}_{2}$ a partir de fontes de combustão. Ainda que, devido às necessidades energéticas da sociedade atual, os combustíveis fósseis ainda estejam sendo usados, ${ }^{184}$ a queima destes compostos gera gases poluentes em excesso, algo que vem contribuindo muito para as mudanças climáticas do planeta. ${ }^{185} \mathrm{~A}$ comunidade científica ultimamente tem se mobilizado para o desenvolvimento de novas matrizes energéticas capazes de substituir estes combustíveis fósseis e que sejam menos poluentes. Pesquisas avançadas indicam que a resposta para essa importante questão talvez esteja em moléculas pequenas e simples como hidrogênio, metano e o gás carbônico (ver Figura 8). ${ }^{186}$

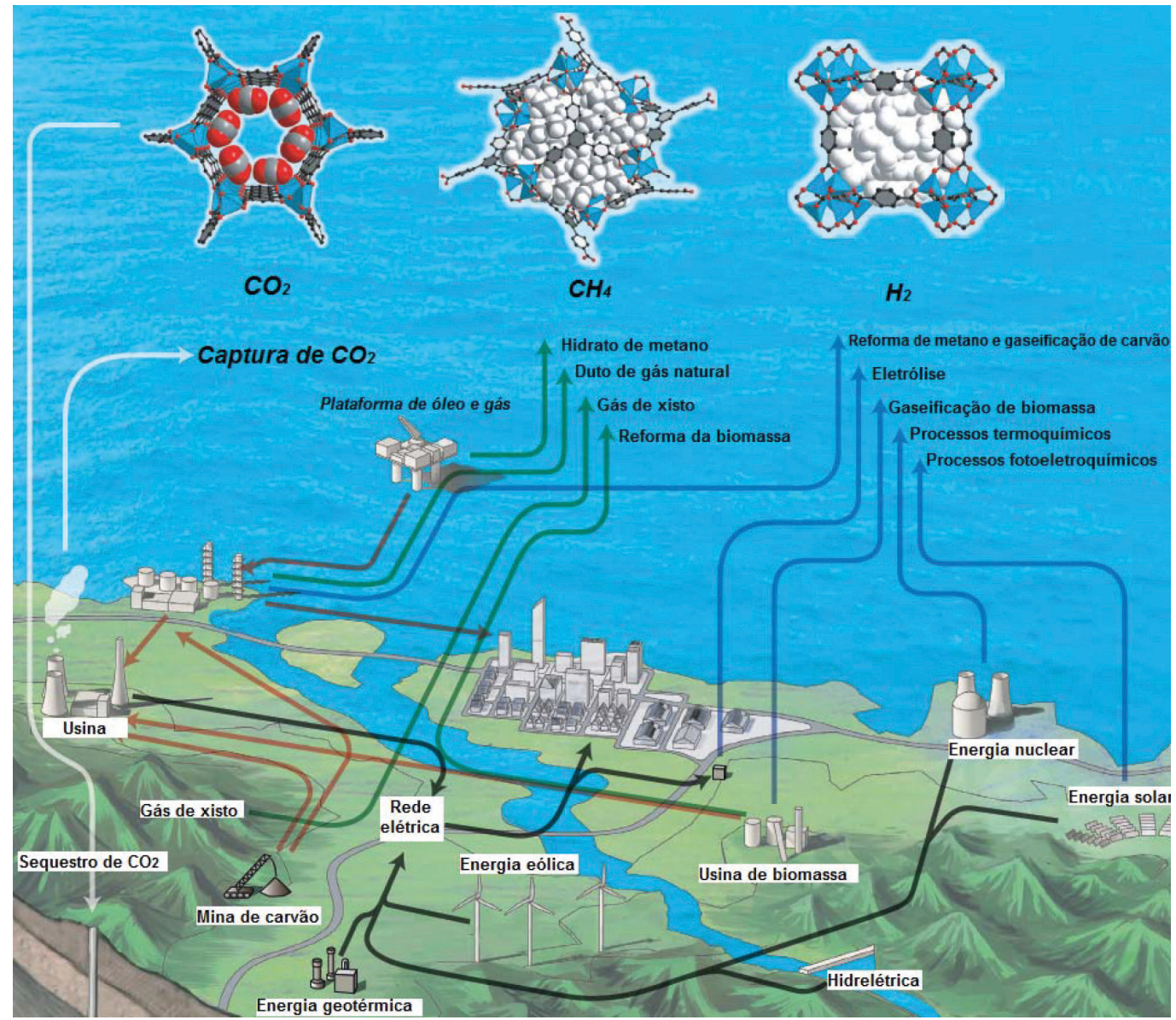

Figura 8. Etapas de produção dos gases essenciais no fornecimento de energia. Setas coloridas representam o fluxo de diferentes vetores energéticos ou sub-produtos: verde, metano; azul, hidrogênio; vermelho, combustíveis tradicionais com alto teor de carbono; preto, eletricidade; branco, dióxido de carbono. Reproduzido da referência 186 com permissão da Nature 
O hidrogênio pode vir a ser uma matriz energética muito eficiente que apresenta água como subproduto de sua combustão. Já o dióxido de carbono e o metano são os principais gases estufa causadores do aquecimento global. Portanto a captura, uso e/ou transformação dessas importantes moléculas começam a despertar o interesse tanto para a obtenção de energia ou compostos industrialmente úteis quanto para reduzir as emissões destes gases na atmosfera. Dentro desse contexto, as MOFs, devido às suas propriedades como elevada área específica, porosidade permanente e possibilidade de funcionalização química, vêm sendo estudadas para utilização na captura e/ou conversão destas pequenas moléculas. ${ }^{186}$ As vantagens no uso destes materiais porosos estão na possibilidade de armazenar gases sem a necessidade de altas pressões, já que eles estarão adsorvidos nos poros da estrutura. Isto torna as MOFs potenciais armazenadoras de gás em células a combustível (vide o protótipo do carro elétrico da Mercedes-Benz F125!) ${ }^{187}$ ou tanques de veículos movidos à hidrogênio ou metano, por exemplo, aumentando a autonomia e a segurança e diminuindo o impacto ambiental (ver a seguir). ${ }^{188}$

Hidrogênio. Devido ao baixo ponto de ebulição e baixa densidade do hidrogênio, o emprego das técnicas normalmente utilizadas para o armazenamento desse gás (compressão em tanques pressurizados ou liquefação em tanques criogênicos) limita o seu transporte, já que os equipamentos são grandes, pesados e apresentam riscos de segurança elevados. ${ }^{189}$ Sendo assim, pesquisas para armazenamento de hidrogênio em materiais porosos como as MOFs tem despertado um grande interesse e se tornado cada vez mais frequentes, visto que a interação com esse gás acontece através do mecanismo de fisissorção, sendo reversível e com uma cinética rápida de adsorção/dessorção. Novos materiais porosos vêm sendo descobertos desde então, sendo necessário que estes possuam poros bem pequenos para ocluir as moléculas de gás hidrogênio e não permitir que elas escapem. A MOF-177, por exemplo, é um composto poroso de zinco que possui área específica de $4.500 \mathrm{~m}^{2} \mathrm{~g}^{-1} \mathrm{e}$ tem sido considerada um dos materiais mais eficientes para adsorção e armazenagem de hidrogênio. ${ }^{189-194}$ Porém, a quantidade de hidrogênio retido à temperatura ambiente ainda é pequena, motivo pelo qual algumas estratégias (funcionalização de ligantes, introdução de sítios metálicos abertos, etc) estão sendo utilizadas para aumentar a eficiência de armazenamento nessa condição. ${ }^{189,195,196}$ A incorporação de nanopartículas metálicas, por exemplo, na estrutura de MOFs está sendo utilizada como uma estratégia para aumentar a força de interação entre as moléculas de hidrogênio e o compósito NPs@MOF sintetizado. Apesar do mecanismo de adsorção não ser completamente conhecido (sugere-se que ocorra através do hydrogen spillover effect, formação de hidrogênio monoatômico pela sua dissociação catalisada), essa abordagem é empregada devido ao forte potencial de adsorção das nanopartículas, à elevada área específica das MOFs e a indicação da ocorrência de uma ligação mais forte com o hidrogênio. ${ }^{105,197}$ Dentro desse contexto, o aumento da eficiência na adsorção de hidrogênio pode ser observado no compósito Pd@ MIL-100(Al), no qual nanopartículas de paládio foram incorporadas na estrutura da MOF de alumínio. Os experimentos mostraram que embora tenha havido uma redução na área específica do material, uma maior adsorção de $\mathrm{H}_{2}$ foi observada à temperatura ambiente. ${ }^{198}$ Além do armazenamento de hidrogênio, MOFs também estão sendo aplicadas para a separação de gases, através de sua utilização na produção de membranas ${ }^{199}$ e filmes. ${ }^{200}$ De fato, o hidrogênio é empregado em muitos processos industriais, sendo necessário realizar sua separação de outros gases. E nesse caso, as propriedades características desses materiais porosos permitem a sua utilização na separação de diversas misturas gasosas envolvendo $\mathrm{H}_{2}$ e $\mathrm{CO}_{2}, \mathrm{H}_{2} \mathrm{O}$ e $\mathrm{CH}_{4}$, por exemplo. ${ }^{201}$

Metano. Embora a energia proveniente do hidrogênio seja limpa, acredita-se que a humanidade ainda dependerá dos combustíveis fósseis por bastante tempo. ${ }^{202}$ Sendo assim, é necessário que haja um controle das emissões de gases estufa para desacelerar o processo de aquecimento global. O metano e o gás carbônico são as principais moléculas responsáveis pelo aumento da temperatura no planeta. A emissão de $1 \mathrm{~kg}$ de $\mathrm{CH}_{4}$ equivale à emissão de $25 \mathrm{~kg}$ de $\mathrm{CO}_{2}$, tornando-o ainda mais poluente. ${ }^{203} \mathrm{~A}$ molécula de $\mathrm{CH}_{4}$, porém, é extremamente energética (entalpia padrão de combustão $=-890 \mathrm{~kJ} \cdot \mathrm{mol}^{-1}$ ) e acredita-se que antes do uso do hidrogênio como matriz energética, o metano será o principal combustível utilizado nos automóveis, sendo este já comercializado com o nome de gás natural. Estudos recentes na área de MOFs visam utilizá-las para armazenamento deste gás para posterior conversão em $\mathrm{CO}_{2}$ através da queima, com o aproveitamento da energia por um automóvel, por exemplo. Existem várias MOFs relatadas na literatura como boas adsorvedoras de metano ${ }^{204-206}$ sendo alguns exemplos os materiais HKUST-1, ${ }^{207-209}$ MOF-519, MOF520, ${ }^{210}$ UTSA-20, ${ }^{211}$ MOF-177, ${ }^{22}$ NOTT-101, ${ }^{212}$ UTSA-110a $^{213}$ e Al-soc-MOF. ${ }^{214}$ Estes sólidos de coordenação porosos vêm apresentando melhores desempenhos frente aos materiais convencionais para armazenamento de $\mathrm{CH}_{4}$ principalmente pelo fato de poderem ser quimicamente modificados afim de aumentar seu potencial de adsorção (ver a seguir). A título de ilustração, a Volkswagen em parceria com a BASF e o grupo do professor Omar Yaghi, desenvolveram um carro protótipo, Caddy EcoFuel car, cujos tanques foram preenchidos com metano adsorvido na Basolite C300 (HKUST-1). Em 2007, um teste foi realizado com esse veículo, em uma viagem que saiu de Berlim em direção à Bangkok na Ásia, resultando em uma autonomia de $32000 \mathrm{Km} !^{215}$ Recentemente duas novas abordagens vêm ganhando a atenção dos pesquisadores na tentativa de aumentar a adsorção de gases como o metano. A primeira estratégia, ilustrada na Figura 9, faz uso de conectores orgânicos topologicamente equivalentes, mas com funcionalidades distintas para a construção de MOFs multivariadas (MTV-MOFs). Dentro deste contexto, Li e colaboradores ${ }^{216}$ construíram MTV-MOFs a partir da NOTT-101 conectando a essa estrutura, ligantes orgânicos contendo os grupamentos piridina, piridazina e pirimidina. Os resultados evidenciaram que o armazenamento de metano pode ser consideravelmente aumentado na presença desses grupos funcionais. A segunda abordagem visa promover a funcionalização pós-sintética de MOFs com grupamentos sítio-específicos que auxiliem na adsorção do gás metano. ${ }^{217,218}$

Uso do biogás como fonte de metano. Uma etapa crítica da utilização do biogás como combustível renovável é a sua purificação, que consiste basicamente na remoção de dióxido de carbono. ${ }^{220}$ Nesse aspecto, a adsorção torna-se uma alternativa viável para a remoção de $\mathrm{CO}_{2}$ a partir do biogás, sendo que diferentes tipos de materiais como argilas, ${ }^{221}$ zeólitas, nanotubos de carbono ${ }^{222}$ e sílicas ${ }^{223}$ vêm sendo estudados para tal fim. E mais recentemente, as MOFs vêm ganhando destaque nesse campo, ${ }^{224,225}$ principalmente quando usadas na forma de membranas. ${ }^{226-228}$ Em um trabalho recente, Ferreira e colaboradores, utilizando a técnica de adsorção por pressão oscilante, estudaram a aplicação da MOF-53(Al) na purificação de misturas de $\mathrm{CO}_{2} / \mathrm{CH}_{4} \cdot{ }^{229}$ Os autores observaram que essa MOF possui uma alta seletividade para $\mathrm{CO}_{2}$ e uma capacidade de adsorção de 4,3 mol kg-1 a uma pressão de 3,5 bar. Além disso, foi realizada a simulação e otimização computacional de dois processos de purificação de biogás em escala industrial, obtendo-se capacidades de recuperação de $\mathrm{CH}_{4}$ de até $93 \%$, além de uma produtividade de $2,78 \mathrm{~mol} \mathrm{~kg}^{-1} \mathrm{~h}^{-1}$. No caso de membranas de matriz mista (MMM), Cheng e colaboradores, utilizando a técnica de spin coating, sintetizaram MMM baseadas em polímeros com microporosidade intrínseca contendo nanofolhas 2D da MOF CuBDC. ${ }^{230} \mathrm{~A}$ otimização da síntese levou à produção de MMM ultrafinas com espessura de $660 \mathrm{~nm}$ e $10 \%$ em massa de CuBDC. As membranas apresentaram uma seletividade de 15,6 para 


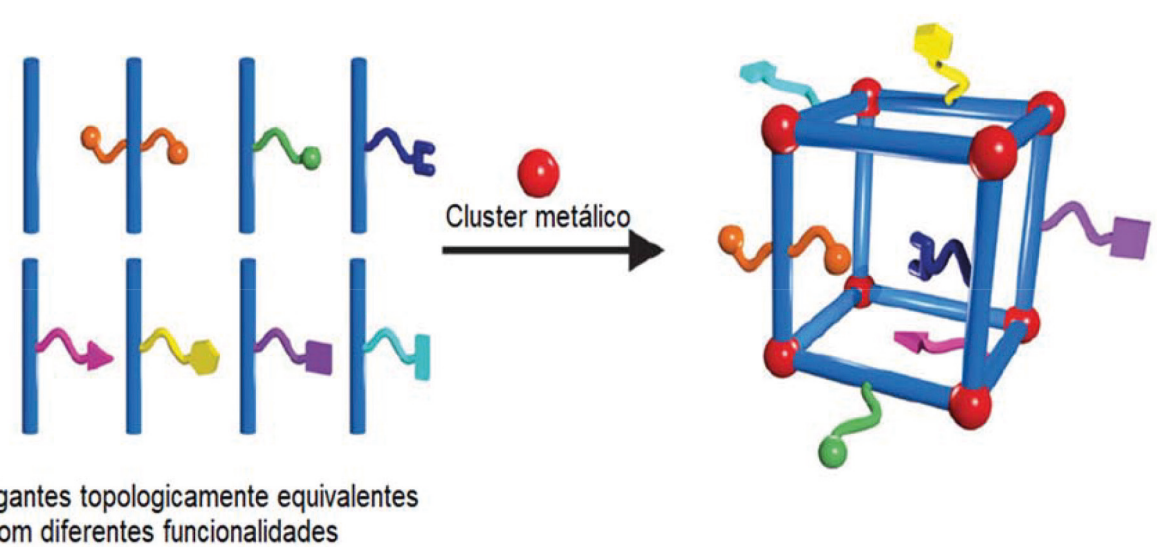

Figura 9. Esquema de construção das MTV-MOFs: integração de diferentes blocos de construção (conectores orgânicos) com tamanho e forma semelhantes, mas com funcionalidades distintas. Reproduzido da referência 219, publicado pela The Royal Society of Chemistry

$\mathrm{CO}_{2} / \mathrm{CH}_{4}$ e uma permeabilidade a $\mathrm{CO}_{2}$ de $407 \mathrm{GPU}$, mostrando-se um promissor material a ser utilizado na purificação de biogás.

Dióxido de carbono. MOFs também têm sido objeto de estudo para captura seletiva de $\mathrm{CO}_{2}$ e como na maioria dos materiais porosos, a adsorção depende da área específica e do tamanho e volume de poros. ${ }^{231}$ Há uma série de pesquisas relatando estes materiais como bons capturadores de gás carbônico ${ }^{232-237}$ sendo recomendável existir sítios abertos (coordenativamente insaturados) nos metais ou grupos funcionais que induzam a polarizabilidade da molécula apolar de $\mathrm{CO}_{2}$. Alguns exemplos são a Mg-MOF-74, ${ }^{238} \mathrm{Cu}$-TDPAT, ${ }^{239}$ SIFSIX-Cu2-I ${ }^{240}$ e MOF-210. ${ }^{22}$

Cabe destacar, no entanto, que a pesquisa atual nessa área tem avançado no sentido de usar as MOFs não apenas para capturar, mas também converter $\mathrm{CO}_{2}$ em produtos químicos de alto valor agregado. ${ }^{241}$ Pesquisas recentes têm mostrado, por exemplo, o uso de MOFs modificadas $^{242}$ ou compósitos para a fotoredução catalítica ${ }^{243,244} \mathrm{de}$ $\mathrm{CO}_{2}{ }^{245-248} \mathrm{~A}$ ideia consiste em realizar fotossíntese artificial para a conversão de $\mathrm{CO}_{2}$ e $\mathrm{H}_{2} \mathrm{O}$ em produtos de maior valor agregado ${ }^{249,250}$ como $\mathrm{CO}, \mathrm{HCOOH}, \mathrm{HCHO}, \mathrm{CH}_{3} \mathrm{OH}$ e $\mathrm{CH}_{4}$. Embora estes estudos ainda estejam em fase inicial, as MOFs $\mathbf{N H}_{2}-\mathbf{M I L - 1 2 5}$ (Ti), $\mathbf{N H}_{2}$ UiO-66 e NH$_{2}$-MIL-101 (Fe) foram recentemente relatadas como fotocatalisadores para a redução de dióxido de carbono sob irradiação de luz na região do visível. Importante destacar que essas MOFs possuem grupos amina que interagem com a molécula de $\mathrm{CO}_{2}$, aumentando assim a adsorção desse gás. ${ }^{248}$ Ainda neste contexto, o desenvolvimento de filmes finos de MOFs sobre a superfície de nanotubos de $\mathrm{Ti} / \mathrm{TiO}_{2}$ foi relatado pela primeira vez por Cardoso e colaboradores ${ }^{251}$ para a redução fotoeletrocatalítica de $\mathrm{CO}_{2}$ com formação de metanol e etanol, como ilustra a Figura 10.

A MOF ZIF-8 foi selecionada para este propósito por apresentar área específica elevada, excelente estabilidade térmica e química, alta capacidade de quimissorção e ativação ${ }^{252} \mathrm{de}_{\mathrm{CO}_{2}}$ e habilidade para mediar a redução do gás sob irradiação UV em baixos potenciais elétricos. Este trabalho demonstra que a fotoeletrocatálise realizada utilizando compósitos de MOFs sobre a superfícies de nanotubos de $\mathrm{Ti} / \mathrm{TiO}_{2}$ tem aplicações promissoras nos campos de conversão de energia e proteção ambiental.

Água. As alterações climáticas e o aumento do crescimento demográfico ameaçam um dos maiores recursos naturais, a água, essencial para a manutenção e subsistência do ecossistema. ${ }^{253}$ Aproximadamente $70 \%$ da superfície do nosso planeta é composto por água, sendo que $2,5 \%$ é água doce e apenas $0,3 \%$ dessa parcela compõe a porção superficial de água doce presente em rios e lagos. ${ }^{254}$ Segundo a Organização das Nações Unidas para a Educação, Ciência

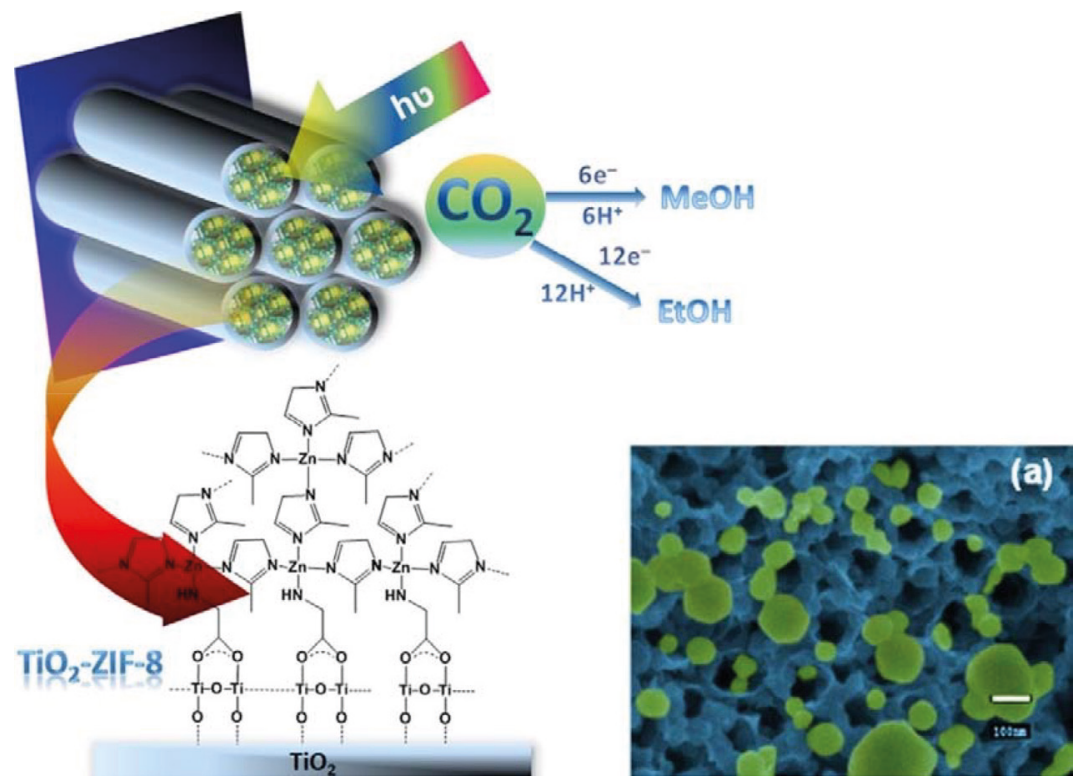

Figura 10. Fotoeletroredução de $\mathrm{CO}_{2}$ catalisada por nanopartículas de $\mathbf{Z I F - 8}$ suportadas sobre nanotubos de Ti/TiO ${ }_{2}$. Reproduzido da referência 251 com permissão da Elsevier 
A

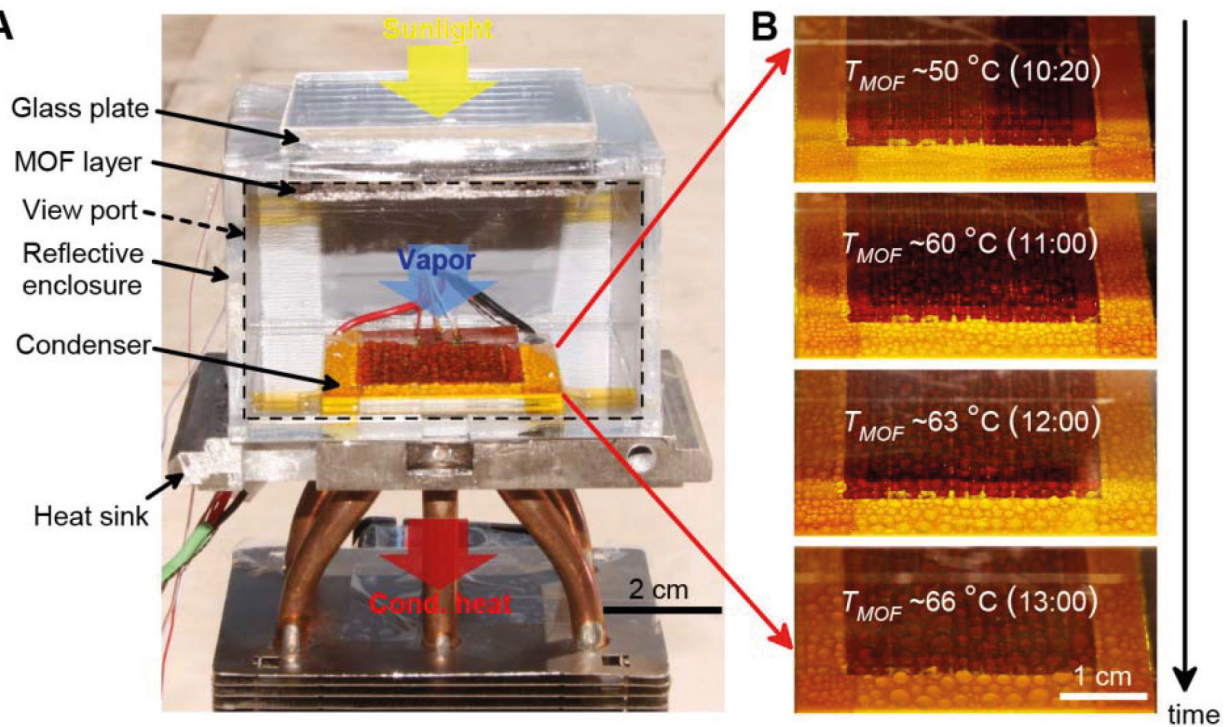

Figura 11. Protótipo do dispositivo de adsorção de água utilizando a MOF-801. Reproduzido da referência 259 com permissão da American Association for the Advancement of Science

e Cultura (Unesco), estima-se para o ano de 2050, uma população mundial entre 9,4 e 10,2 bilhões de pessoas e um aumento de 55\% na demanda de água (entre consumo, higiene, alimentação e energia). Nessa projeção, e esse é um dado alarmante, mais de $45 \%$ da população mundial não atingirá a cota diária mínima de 50 litros de água por pessoa. ${ }^{255}$ Nesse contexto, a adsorção de moléculas de água em materiais porosos pode ser uma solução promissora frente aos atuais geradores de água atmosférica que consomem muita energia para condensação do vapor. ${ }^{256}$ Dentre esses materiais, merecem destaque as MOFs de zircônio MOF-801 e MOF-841, que adsorvem cerca de $32 \%$ e $40 \%$ de água em massa, respectivamente, ${ }^{257}$ e a IRMOF-74 contendo cobalto como centro metálico, que sob simulações de condições desérticas $45{ }^{\circ} \mathrm{C}$, umidade relativa de $5 \%$ durante o dia e $35 \%$ à noite., é capaz de armazenar $82 \%$ de água em massa. ${ }^{258}$ Visando, então, comprovar a eficiência de adsorção em condições reais, Yaghi e colaboradores desenvolveram um aparato baseado na MOF-801, $\left.\left[\mathrm{Zr}_{6} \mathrm{O}_{4}(\mathrm{OH})_{4} \text { (fumarato }\right)_{6}\right]$, que foi capaz de extrair água potável do ar no deserto de Tempe, no Arizona, USA. O protótipo do aparato está ilustrado na Figura 11. Durante o período da noite, a umidade atmosférica (tão baixa quanto 20\%) é adsorvida pela MOF e o processo de dessorção ocorre durante o dia, com o calor gerado pelo sol. O vapor dessorvido é, então, condensado e coletado (cerca de $0,28 \mathrm{~L}$ de água por quilograma de MOF por dia). ${ }^{259}$ Ainda que sejam resultados preliminares e o experimento tenha sido realizado em uma escala muito pequena, o desenvolvimento de novas MOFs com altas capacidade e cinética de adsorção pode levar a um aumento significativo na extração de água líquida do vapor atmosférico. ${ }^{260}$

\section{CONSIDERAÇÕES FINAIS}

Esperamos que esse trabalho possa ajudar a despertar o interesse por essa fascinante área de pesquisa, cuja importância tem sido demonstrada não só pelo número muito grande de publicações e o engajamento crescente de pesquisadores de outros campos do conhecimento, como também pelo emergente interesse comercial. As MOFs são materiais versáteis por possuírem um número quase ilimitado de combinações entre metais e ligantes politópicos, e sua porosidade e área específica podem ser controladas em função do método de síntese e da natureza do ligante utilizado. As demais propriedades como alta cristalinidade, boa estabilidade térmica e possibilidade de modificações estruturais que facilitam o reconhecimento de determinados grupos funcionais em regiões sítio-específicas contribuem para a vasta gama de aplicações em potencial. Dentro deste contexto, as MOFs vêm contribuindo para a solução de problemas ambientais, relacionados a quatro pequenas, porém importantes, moléculas: hidrogênio, metano, dióxido de carbono e água. O uso de MOFs para armazenamento de hidrogênio e metano pode ser uma potencial aplicação para substituir combustíveis fósseis. As altas capacidades de adsorção desses materiais podem também ser aproveitadas para captura de dióxido de carbono, visando a transformação deste gás em materiais combustíveis (metano e metanol) e/ou compostos de alto valor agregado (ácido fórmico, formaldeído), minimizando os impactos decorrentes do efeito estufa. Na adsorção de água, a MOF$\mathbf{8 0 1}$ tem auxiliado na captação de água líquida a partir da umidade atmosférica, sem gasto e utilização de energia elétrica, na tentativa de solucionar problemas relacionados a sua escassez em condições extremas, como as desérticas. Concluindo, novas MOFs com propriedades ainda inimagináveis deverão ser descobertas e, por ser uma área relativamente nova, ainda há muito a ser estudado. É a intenção dos autores desse trabalho que ele possa de alguma forma motivar o surgimento de novos grupos de pesquisa em MOFs no Brasil.

\section{AGRADECIMENTOS}

Os autores agradecem a CAPES, ao CNPq e a FAPESP pelo apoio recebido.

\section{REFERÊNCIAS}

1. Li, H.; Eddaoudi, M.; O'Keeffe, M.; Yaghi, O. M.; Nature 1999, 402, 276.

2. Batten, S. R.; Champness, N. R.; Chen, X.-M.; Garcia-Martinez, J.; Kitagawa, S.; Öhrström, L.; O'Keeffe, M.; Suh, M. P.; Reedijk, J.; CrystEngComm 2012, 14, 3001.

3. Levason, B.; Bradshaw, D.; Coord. Chem. Rev. 2016, 307, 105.

4. Coudert, F. X.; Fuchs, A. H.; Coord. Chem. Rev. 2015, 307, 211.

5. Kaskel, S.; Fischer, R.; J. Mater. Chem. 2012, 22, 10093.

6. Li, M.; Li, D.; O'Keeffe, M.; Yaghi, O. M. ;Chem. Rev. 2014, 114, 1343.

7. Yuan, S.; Feng, L.; Wang, K.; Pang, J.; Bosch, M.; Lollar, C.; Sun, Y.; Qin, J.; Yang, X.; Zhang, P.; Wang, Q.; Zou, L.; Zhang, Y.; Zhang, L.; Fang, Y.; Li, J.; Zhou, H. C.; Adv. Mater. 2018, 1704303, 1.

8. Kaskel, S.; The Chemistry of Metal-Organic Frameworks. Synthesis, 
Characterization and Applications, Wiley: New York, 2016.

9. Jiang, J.; Metal-Organic Frameworks Materials Modeling towards Potential Engineering Applications, Pan Stanford Publ.: Florida, 2015.

10. MacGillivray, L. R.; Lukehart, C. M.; Metal-Organic Framework Materials, Wiley-VCH Verlag: Berlin, 2014.

11. Farrusseng, D.; Metal-Organic Frameworks. Applications from Catalysis to Gas Storage, Wiley-VCH Verlag: Berlin, 2011.

12. Schröder, M.; Functional Metal-Organic Frameworks: Gas Storage, Separation and Catalysis, Springer: Berlin, 2010.

13. Frem, R. C. G.; Arroyos, G.; Lucena, G. N.; Flor, J. B. S.; Fávaro, M. A.; Coura, M. F.; Alves, R. C. In Recent Advances in Complex Functional Materials From Design to Application; Longo, E., La Porta, F. A., eds.; Springer: Berlin, 2017, cap. 14.

14. Sumida, K.; Arnold, J.; J. Chem. Educ. 2011, 88, 92.

15. Crane, J. L.; Anderson, K. E.; Conway, S. G.; J. Chem. Educ. 2015, 92, 373.

16. Brown, T. E.; LeMay, H. E.; Bursten, B. E.; Murphy, C.; Woodward, P.; Stoltzfus, M. E.; Chemistry: The Central Science, $13^{\text {th }}$ ed., Pearson: London, 2015.

17. Dantas Ramos, A. L.; Tanase, S.; Rothenberg, G.; Quim. Nova 2014, 37, 123.

18. Galaço, A. R. B. S.; Lima, J. F.; Serra, O. A.; Quim. Nova 2018, 41, 678.

19. Alves-Jr, S.; Metal Organic Frameworks (MOFs): da síntese a aplicações em meio ambiente, saúde e segurança pública, CRV Ltda.: Curitiba, 2016.

20. Huskic, I.; Pekov, I. V.; Krivovichev, S. V.; Friscic, T.; Sci. Adv. 2016, 2 , e1600621.

21. Farha, O. K.; Eryazici, I.; Jeong, N. C.; Hauser, B. G.; Wilmer, C. E.; Sarjeant, A. A.; Snurr, R. Q.; Nguyen, S. T.; Yazaydin, A. Ö.; Hupp, J. T.; J. Am. Chem. Soc. 2012, 134, 15016.

22. Furukawa, H.; Ko, N.; Go, Y. B.; Aratani, N.; Choi, S. B.; Choi, E.; Yazaydin, A. O.; Snurr, R. Q.; O’Keeffe, M.; Kim, J.; Yaghi, O. M.; Science 2010, 329, 424

23. Song, Z.; Nambo, A.; Tate, K. L.; Bao, A.; Zhu, M.; Jasinski, J. B.; Zhou, S. J.; Meyer, H. S.; Carreon, M. A.; Li, S.; Yu, M.; Nano Lett. 2016, 16, 3309.

24. Férey, G.; Serre, C.; Devic, T.; Maurin, G.; Jobic, H.; Llewellyn, P. L.; De Weireld, G.; Vimont, A.; Daturi, M.; Chang, J.-S.; Chem. Soc. Rev. 2011, 40, 550.

25. Férey, G.; Serre, C.; Chem. Soc. Rev. 2009, 38, 1380.

26. Llabrés i Xamena, F. X.; Gascon, J.; Metal Organic Frameworks as Heterogeneous Catalysts, RSC Publ.: London, 2013.

27. Erucar, I.; Keskin, S.; Ind. Eng. Chem. Res. 2016, 55, 1929.

28. Mingos, D. M. P. In Metal-Organic Frameworks for Photonics Applications; Chen, B., Qian, G., eds.; Springer: Berlin, 2014.

29. Zou, Z.; Wang, S.; Jia, J.; Xu, F.; Long, Z.; Hou, X.; Microchem. J. 2016, 124, 578.

30. Choi, K. M.; Jeong, H. M.; Park, J. H.; Zhang, Y.; Kang, J. K.; Yaghi, O. M.; ACS Nano 2014, 8, 7451 .

31. Yamagiwa, H.; Sato, S.; Fukawa, T.; Ikehara, T.; Maeda, R.; Mihara, T.; Kimura, M.; Sci. Rep. 2015, 4, 6247.

32. Coronado, E.; Mínguez Espallargas, G.; Chem. Soc. Rev. 2013, 42, 1525.

33. Batten, S. R.; Champness, N. R.; Chen, X.-M.; Garcia-Martinez, J.; Kitagawa, S.; Öhrström, L.; O’ Keeffe, M.; Suh, M. P.; Reedijk, J.; Pure Appl. Chem. 2013, 85, 1715.

34. Yukio, K.; Ikuo, M.; Taiichi, H.; Yoshihiko, S.; Bull. Chem. Soc. Jpn. 1959, 32, 1221.

35. Hoskins, B. F.; Robson, R.; J. Am. Chem. Soc. 1989, 111, 5962.

36. Wells, A. F.; Bull. Am. Math. Soc. 1978, 84, 466.

37. Li, H.; Eddaoudi, M.; Groy, T. L.; Yaghi, O. M.; J. Am. Chem. Soc. 1998, $120,8571$.

38. Ferey, G.; Mellot-Draznieks, C.; Serre, C.; Millange, F.; Dutour, J.; Science 2005, 309, 2040.
39. Chui, S.-Y. S.; Lo, S. M.-F.; Charmant, J. P. H.; Orpen, A. G.; Williams, I. D.; Science 1999, 283, 1148.

40. Kandiah, M.; Nilsen, M. H.; Usseglio, S.; Jakobsen, S.; Olsbye, U.; Tilset, M.; Larabi, C.; Quadrelli, E. A.; Bonino, F.; Lillerud, K. P.; Chem. Mater. 2010, 22, 6632.

41. Michl, J. Modular Chemistry, Kluwer Academic Publishers: Dordrecht, 1995.

42. Yaghi, O. M.; Li, H.; Davis, C.; Richardson, D.; Groy, T. L.; Acc. Chem. Res. 1998, 31, 474.

43. Eddaoudi, M.; Moler, D. B.; Li, H.; Chen, B.; Reineke, T. M.; O'Keeffe, M.; Yaghi, O. M.; Acc. Chem. Res. 2001, 34, 319.

44. Yaghi, O. M.; O'Keeffe, M.; Ockwig, N. W.; Chae, H. K.; Eddaoudi, M.; Kim, J.; Nature 2003, 423, 705.

45. Cook, T. R.; Zheng, Y. R.; Stang, P. J.; Chem. Rev. 2013, 113, 734.

46. Yaghi, O. M.; Li, Q.; MRS Bull. 2009, 34, 682.

47. Eddaoudi, M.; Kim, J.; O'Keeffe, M.; Yaghi, O. M.; J. Am. Chem. Soc. 2002, 124, 376

48. O'Keeffe, M.; Eddaoudi, M.; Li, H.; Reineke, T.; Yaghi, O. M.; J. Solid State Chem. 2000, 152, 3.

49. Ockwig, N. W.; Delgado-Friedrichs, O.; O’Keeffe, M.; Yaghi, O. M.; Acc. Chem. Res. 2005, 38, 176

50. http://rcsr.anu.edu.au, acessada em agosto de 2018.

51. O'Keeffe, M.; Peskov, M. A.; Ramsden, S. J.; Yaghi, O. M.; Acc. Chem. Res. 2008, 41, 1782.

52. Hendon, C. H.; Walsh, A.; Chem. Sci. 2015, 6, 3674.

53. Öhrström, L.; Crystals 2015, 5, 154.

54. Eubank, J. F.; Walsh, R. D.; Eddaoudi, M.; Chem. Commun. 2005, 0, 2095.

55. Biradha, K.; Fujita, M.; Angew. Chem., Int. Ed. 2002, 41, 3392.

56. Dietzel, P. D. C.; Morita, Y.; Blom, R.; Fjellvåg, H.; Angew. Chem., Int. Ed. 2005, 44, 6354

57. Furukawa, H.; Go, Y. B.; Ko, N.; Park, Y. K.; Uribe-Romo, F. J.; Kim, J.; O'Keeffe, M.; Yaghi, O. M.; Inorg. Chem. 2011, 50, 9147.

58. Furukawa, H.; Kim, J.; Ockwig, N. W.; O’Keeffe, M.; Yaghi, O. M.; J. Am. Chem. Soc. 2008, 130, 11650.

59. Liang, L.-L.; Zhang, J.; Ren, S.-B.; Ge, G.-W.; Li, Y.-Z.; Du, H.-B.; You, X.-Z.; CrystEngComm 2010, 12, 2008.

60. Noro, S.; Kitagawa, S.; Kondo, M.; Seki, K.; Angew. Chem., Int. Ed. 2000, 39, 2081

61. Furukawa, H.; Cordova, K. E.; O'Keeffe, M.; Yaghi, O. M.; Science 2013, 341, 1230444.

62. Lu, J.; Mondal, A.; Moulton, B.; Zaworotko, M. J.; Angew. Chem., Int. Ed. 2001, 113, 2171.

63. Perry IV, J. J.; Perman, J. A.; Zaworotko, M. J.; Chem. Soc. Rev. 2009 , 38,1400 .

64. Zaworotko, M. J.; Nat. Chem. 2009, 1, 267.

65. Park, J.; Hong, S.; Moon, D.; Park, M.; Lee, K.; Kang, S.; Zou, Y.; John, R. P.; Kim, G. H.; Lah, M. S.; Inorg. Chem. 2007, 46, 10208.

66. Guillerm, V.; Kim, D.; Eubank, J. F.; Luebke, R.; Liu, X.; Adil, K.; Lah, M. S.; Eddaoudi, M.; Chem. Soc. Rev. 2014, 43, 6141.

67. Kim, D.; Liu, X.; Lah, M. S.; Inorg. Chem. Front. 2015, 2, 336.

68. Liu, J.; Chen, L.; Cui, H.; Zhang, J.; Zhang, L.; Su, C.-Y.; Chem. Soc. Rev. 2014, 43, 6011.

69. Yaghi, O. M.; Li, G.; Li, H.; Nature 1995, 378, 703.

70. Sanda, S.; Parshamoni, S.; Konar, S.; Inorg. Chem. 2013, 52, 12866.

71. Serre, C.; Bourrelly, S.; Vimont, A.; Ramsahye, N. A.; Maurin, G.; Llewellyn, P. L.; Daturi, M.; Filinchuk, Y.; Leynaud, O.; Barnes, P.; Férey, G.; Adv. Mater. 2007, 19, 2246.

72. Wang, Z.; Cohen, S. M.; Chem. Soc. Rev. 2009, 38, 1315.

73. Cohen, S. M.; Chem. Rev. 2012, 112, 970.

74. Burrows, A. D.; Frost, C. G.; Mahon, M. F.; Richardson, C.; Angew. Chem., Int. Ed. 2008, 47, 8482.

75. Hu, Z.; Zhao, D.; Dalton Trans. 2015, 44, 19018. 
76. Seoane, B.; Castellanos, S.; Dikhtiarenko, A.; Kapteijn, F.; Gascon, J.; Coord. Chem. Rev. 2016, 307, 147.

77. Dey, C.; Kundu, T.; Biswal, B. P.; Mallick, A.; Banerjee, R.; Acta Crystallogr., Sect. B: Struct. Sci., Cryst. Eng. Mater. 2014, 70, 3.

78. Stock, N.; Biswas, S.; Chem. Rev. 2012, 112, 933.

79. He, M.; Yao, J.; Liu, Q.; Wang, K.; Chen, F.; Wang, H.; Microporous Mesoporous Mater. 2014, 184, 55.

80. Williams, I. D.; Nat. Chem. 2014, 6, 953.

81. Klinowski, J.; Almeida Paz, F. A.; Silva, P.; Rocha, J.; Dalton Trans. 2011, 40, 321.

82. Son, W.-J.; Kim, J.; Kim, J.; Ahn, W.-S.; Chem. Commun. 2008, 47, 6336.

83. Martinez-Joaristi, A.; Juan-Alcañiz, J.; Serra-Crespo, P.; Kapteijn, F.; Gascon, J.; Cryst. Growth Des. 2012, 12, 3489.

84. Stassen, I.; Styles, M.; Van Assche, T.; Campagnol, N.; Fransaer, J.; Denayer, J.; Tan, J. C.; Falcaro, P.; De Vos, D.; Ameloot, R.; Chem. Mater. 2015, 27, 1801.

85. Sakamoto, H.; Matsuda, R.; Kitagawa, S.; Dalton Trans. 2012, 41, 3956.

86. Friščić, T.; J. Mater. Chem. 2010, 20, 7599.

87. Pichon, A.; Lazuen-Garay, A.; James, S. L.; CrystEngComm 2006, 8, 211.

88. Zhan, G.; Zeng, H. C.; Chem. Commun. 2017, 53, 72.

89. Shi, Q.; Chen, Z.; Song, Z.; Li, J.; Dong, J.; Angew. Chem., Int. Ed. 2011, 50, 672 .

90. Ahmed, I.; Jeon, J.; Khan, N. A.; Jhung, S. H.; Cryst. Growth Des. 2012, $12,5878$.

91. Das, A. K.; Vemuri, R. S.; Kutnyakov, I.; McGrail, B. P.; Motkuri, R. K.; Sci. Rep. 2016, 6, 28050.

92. Mueller, U.; Puetter, H.; Hesse, M.; Wessel, H.; WO 2005/049892 2015.

93. Gimeno-Fabra, M.; Munn, A. S.; Stevens, L. A.; Drage, T. C.; Grant, D. M.; Kashtiban, R. J.; Sloan, J.; Lester, E.; Walton, R. I.; Chem. Commun. 2012, 48, 10642

94. Rubio-Martinez, M.; Batten, M. P.; Polyzos, A.; Carey, K.-C.; Mardel, J. I.; Lim, K.-S.; Hill, M. R.; Sci. Rep. 2014, 4, 1.

95. Dunne, P. W.; Munn, A. S.; Starkey, C. L.; Huddle, T. A.; Lester, E. H.; Philos. Trans. R. Soc., A 2015, 373, 20150015.

96. McKinstry, C.; Cathcart, R. J.; Cussen, E. J.; Fletcher, A. J.; Patwardhan, S. V.; Sefcik, J.; Chem. Eng. J. 2016, 285, 718.

97. Garzón-Tovar, L.; Cano-Sarabia, M.; Carné-Sánchez, A.; Carbonell, C.; Imaz, I.; Maspoch, D.; React. Chem. Eng. 2016, 1, 533.

98. Garcia Marquez, A.; Horcajada, P.; Grosso, D.; Ferey, G.; Serre, C.; Sanchez, C.; Boissiere, C.; Chem. Commun. 2013, 49, 3848.

99. Crawford, D.; Casaban, J.; Haydon, R.; Giri, N.; McNally, T.; James, S. L.; Chem. Sci. 2015, 6, 1645.

100. Reinsch, H.; Waitschat, S.; Chavan, S. M.; Lillerud, K. P.; Stock, N.; Eur. J. Inorg. Chem. 2016, 2016, 4490.

101. Majano, G.; Pérez-Ramírez, J.; Adv. Mater. 2013, 25, 1052.

102. Huo, J.; Brightwell, M.; El Hankari, S.; Garai, A.; Bradshaw, D.; J. Mater. Chem. A 2013, 1, 15220.

103. Cho, H. Y.; Kim, J.; Kim, S. N.; Ahn, W. S.; Microporous Mesoporous Mater. 2013, 169, 180.

104. Chen, Y.; Huang, X.; Zhang, S.; Li, S.; Cao, S.; Pei, X.; Zhou, J.; Feng, X.; Wang, B.; J. Am. Chem. Soc. 2016, 138, 10810.

105. Ren, J.; Musyoka, N. M.; Langmi, H. W.; Swartbooi, A.; North, B. C.; Mathe, M.; Int. J. Hydrogen Energy 2015, 40, 4617.

106. Moitra, N.; Fukumoto, S.; Reboul, J.; Sumida, K.; Zhu, Y.; Nakanishi, K.; Furukawa, S.; Kitagawa, S.; Kanamori, K.; Chem. Commun. 2015 , $51,3511$.

107. Furukawa, S.; Reboul, J.; Diring, S.; Sumida, K.; Kitagawa, S.; Chem. Soc. Rev. 2014, 43, 5700.

108. Lee, J.; Kwak, J. H.; Choe, W.; Nat. Commun. 2017, 8, 14070.

109. Czaja, A. U.; Trukhan, N.; Müller, U.; Chem. Soc. Rev. 2009, 38, 1284.
110. Silva, P.; Vilela, S. M. F.; Tomé, J. P. C.; Almeida Paz, F. A.; Chem. Soc. Rev. 2015, 44, 6774.

111. Zacher, D.; Schmid, R.; Wöll, C.; Fischer, R. A.; Angew. Chem., Int. Ed. 2011, 50, 176 .

112. Li, W.-J.; Liu, J.; Sun, Z.-H.; Liu, T.-F.; Lü, J.; Gao, S.-Y.; He, C.; Cao, R.; Luo, J.-H.; Nat. Commun. 2016, 7, 11830.

113. Stassen, I.; Styles, M.; Grenci, G.; Gorp, H. V.; Vanderlinden, W.; Feyter, S. D.; Falcaro, P.; Vos, D. De; Vereecken, P.; Ameloot, R.; Nat. Mater. 2015, 15, 304 .

114. Qiu, S.; Xue, M.; Zhu, G.; Chem. Soc. Rev. 2014, 43, 6116.

115. Kong, L.; Zhang, X.; Liu, Y.; Li, S.; Liu, H.; Qiu, J.; Yeung, K. L.; Mater. Chem. Phys. 2014, 148, 10.

116. Aguilera-Sigalat, J.; Bradshaw, D.; Coord. Chem. Rev. 2015, 307, 267.

117. Zhu, Q.-L.; Xu, Q.; Chem. Soc. Rev. 2014, 43, 5468.

118. Chui, S. S. Y.; Lo, S. M. F.; Charmant, J. P. H.; Orpen, a G.; Williams, I. D.; Science 1999, 283, 1148

119. Lin, K. S.; Adhikari, A. K.; Ku, C. N.; Chiang, C. L.; Kuo, H.; Int. J. Hydrogen Energy 2012, 37, 13865.

120. Millange, F.; Medina, M. I.; Guillou, N.; Férey, G.; Golden, K. M.; Walton, R. I.; Angew. Chem., Int. Ed. 2010, 49, 763.

121. Stavitski, E.; Goesten, M.; Juan-Alcañiz, J.; Martinez-Joaristi, A.; SerraCrespo, P.; Petukhov, A. V.; Gascon, J.; Kapteijn, F.; Angew. Chem., Int. Ed. 2011, 50, 9624.

122. Spokoyny, A. M.; Kim, D.; Sumrein, A.; Mirkin, C. A.; Chem. Soc. Rev. 2009, 38, 1218.

123. Carné, A.; Carbonell, C.; Imaz, I.; Maspoch, D.; Chem. Soc. Rev. 2011, 40, 291.

124. Sindoro, M.; Yanai, N.; Jee, A. Y.; Granick, S.; Acc. Chem. Res. 2014, $47,459$.

125. Bloch, E. D.; Queen, W. L.; Krishna, R.; Zadrozny, J. M.; Brown, C. M.; Long, J. R. Science 2012, 335, 1606.

126. Masoomi, M. Y.; Beheshti, S.; Morsali, A.; Cryst. Growth Des. 2015, 15, 2533.

127. Diaz-Garcia, M.; Mayoral, A.; Diaz, I.; Sanchez-Sanchez, M.; Cryst. Growth Des. 2014, 14, 2479.

128. Bunzen, H.; Grzywa, M.; Hambach, M.; Spirkl, S.; Volkmer, D.; Cryst. Growth Des. 2016, 16, 3190.

129. Jin, L.-N.; Liu, Q.; Sun, W.-Y.; CrystEngComm 2014, 16, 3816.

130. Flügel, E. A.; Ranft, A.; Haase, F.; Lotsch, B. V.; J. Mater. Chem. 2012 , $22,10119$.

131. Diring, S.; Furukawa, S.; Takashima, Y.; Tsuruoka, T.; Kitagawa, S.; Chem. Mater. 2010, 22, 4531.

132. Guo, H.; Zhu, Y.; Qiu, S.; Lercher, A. J.; Zhang, H.; Adv. Mater. 2010, 22,4190 .

133. Umemura, A.; Diring, S.; Furukawa, S.; Uehara, H.; Tsuruoka, T.; Kitagawa, S.; J. Am. Chem. Soc. 2011, 133, 15506.

134. Cravillon, J.; Münzer, S.; Lohmeier, S. J.; Feldhoff, A.; Huber, K.; Wiebcke, M.; Chem. Mater. 2009, 21, 1410.

135. Rieter, W. J.; Taylor, K. M. L.; An, H.; Lin, W.; Lin, W.; J. Am. Chem. Soc. 2006, 128, 9024.

136. Liu, Q.; Jin, L.-N.; Sun, W.-Y.; Chem. Commun. 2012, 48, 8814.

137. Carné-Sánchez, A.; Imaz, I.; Cano-Sarabia, M.; Maspoch, D.; Nat. Chem. 2013, 5, 203.

138. Hyo Park, J.; Min Choi, K.; Joon Jeon, H.; Jung Choi, Y.; Ku Kang, J.; Sci. Rep. 2015, 5, 12045.

139. Bradshaw, D.; El-Hankari, S.; Lupica-Spagnolo, L.; Chem. Soc. Rev. 2014, 43, 5431 .

140. Grünker, R.; Bon, V.; Müller, P.; Stoeck, U.; Krause, S.; Mueller, U.; Senkovska, I.; Kaskel, S.; Chem. Commun. 2014, 50, 3450.

141. Song, L.; Zhang, J.; Sun, L.; Xu, F.; Li, F.; Zhang, H.; Si, X.; Jiao, C.; Li, Z.; Liu, S.; Liu, Y.; Zhou, H.; Sun, D.; Du, Y.; Cao, Z.; Gabelica, Z.; Energy Environ. Sci. 2012, 5, 7508.

142. Xuan, W.; Zhu, C.; Liu, Y.; Cui, Y.; Chem. Soc. Rev. 2012, 41, 1677. 
143. Ahmed, A.; Hodgson, N.; Barrow, M.; Clowes, R.; Robertson, C. M.; Steiner, A.; McKeown, P.; Bradshaw, D.; Myers, P.; Zhang, H.; J. Mater. Chem. A 2014, 2, 9085.

144. Li, T.; Kozlowski, M. T.; Doud, E. A.; Blakely, M. N.; Rosi, N. L.; J. Am. Chem. Soc. 2013, 135, 11688.

145. Deng, H.; Grunder, S.; Cordova, K. E.; Valente, C.; Furukawa, H.; Hmadeh, M.; Gandara, F.; Whalley, A. C.; Liu, Z.; Asahina, S.; Kazumori, H.; O'Keeffe, M.; Terasaki, O.; Stoddart, J. F.; Yaghi, O. M.; Science 2012, 336, 1018.

146. Chen, B.; Eddaoudi, M.; Hyde, S. T.; O'Keeffe, M.; Yaghi, O. M.; Science 2001, 291, 1021.

147. Shekhah, O.; Wang, H.; Paradinas, M.; Ocal, C.; Schüpbach, B.; Terfort, A.; Zacher, D.; Fischer, R. A.; Wöll, C.; Nat. Mater. 2009, 8, 481.

148. Farha, O. K.; Malliakas, C. D.; Kanatzidis, M. G.; Hupp, J. T.; J. Am. Chem. Soc 2010, 132, 950.

149. Seoane, B.; Dikhtiarenko, A.; Mayoral, A.; Tellez, C.; Coronas, J.; Kapteijn, F.; Gascon, J.; CrystEngComm 2015, 17, 1693.

150. Zhao, Y.; Zhang, J.; Han, B.; Song, J.; Li, J.; Wang, Q.; Angew. Chem., Int. Ed. 2011, 50, 636.

151. Qiu, L. G.; Xu, T.; Li, Z. Q.; Wang, W.; Wu, Y.; Jiang, X.; Tian, X. Y.; Zhang, L.; Angew. Chem., Int. Ed. 2008, 47, 9487.

152. Li, B.; Chrzanowski, M.; Zhang, Y.; Ma, S.; Coord. Chem. Rev. 2016, 307, 106.

153. Kong, X.; Deng, H.; Yan, F.; Kim, J.; Swisher, J. A.; Smit, B.; Yaghi, O. M.; Reimer, J. A.; Science 2013, 341, 882.

154. Deria, P.; Mondloch, J. E.; Karagiaridi, O.; Bury, W.; Hupp, J. T.; Farha, O. K.; Chem. Soc. Rev. 2014, 43, 5896.

155. Cohen, S. M. Chem. Sci. 2010, 1, 32.

156. Karagiaridi, O.; Bury, W.; Mondloch, J. E.; Hupp, J. T.; Farha, O. K.; Angew. Chem., Int. Ed. 2014, 53, 4530.

157. Evans, J. D.; Sumby, C. J.; Doonan, C. J.; Chem. Soc. Rev. 2014, 43, 5933.

158. Brozek, C. K.; Dincă, M. Chem. Soc. Rev.; 2014, 43, 5456.

159. Han, Y.; Li, J.-R.; Xie, Y.; Guo, G.; Chem. Soc. Rev. 2014, 43, 5952.

160. Huang, L.; He, M.; Chen, B.; Hu, B.; J. Mater. Chem. A 2015, 3, 11587.

161. Juan-Alcañiz, J.; Gascon, J.; Kapteijn, F.; J. Mater. Chem. 2012, 22, 10102.

162. Esken, D.; Turner, S.; Lebedev, O. I.; Van Tendeloo, G.; Fischer, R. A.; Chem. Mater. 2010, 22, 6393.

163. Lu, G.; Li, S.; Guo, Z.; Farha, O. K.; Hauser, B. G.; Qi, X.; Wang, Y.; Wang, X.; Han, S.; Liu, X.; DuChene, J. S.; Zhang, H.; Zhang, Q.; Chen, X.; Ma, J.; Loo, S. C. J.; Wei, W. D.; Yang, Y.; Hupp, J. T.; Huo, F.; Nat. Chem. 2012, 4, 310.

164. Li, B.; Wen, H. M.; Cui, Y.; Zhou, W.; Qian, G.; Chen, B. ;Adv. Mater. 2016, 28, 8819.

165. Bai, Y.; Dou, Y.; Xie, L.-H.; Rutledge, W.; Li, J.-R.; Zhou, H.-C.; Chem. Soc. Rev. 2016, 45, 2327.

166. Cui, Y.; Li, B.; He, H.; Zhou, W.; Chen, B.; Qian, G.; Acc. Chem. Res. 2016, 49, 483.

167. Imaz, I.; Rubio-Martínez, M.; An, J.; Solé-Font, I.; Rosi, N. L.; Maspoch, D.; Chem. Commun. 2011, 47, 7287.

168. Giménez-Marqués, M.; Hidalgo, T.; Serre, C.; Horcajada, P.; Coord. Chem. Rev. 2015, 307, 342.

169. Tan, L.-L.; Song, N.; Zhang, S. X.-A.; Li, H.; Wang, B.; Yang, Y.-W.; J. Mater. Chem. B 2016, 4, 135.

170. Doonan, C.; Riccò, R.; Liang, K.; Bradshaw, D.; Falcaro, P.; Acc. Chem. Res. 2017, 50, 1423.

171. Lahoud, M. G.; Frem, R. C. G.; Marques, L. F.; Arroyos, G.; Brandão, P.; Ferreira, R. A. S.; Carlos, L. D.; J. Solid State Chem. 2017, 253.

172. Stavila, V.; Talin, A. A.; Allendorf, M. D.; Chem. Soc. Rev. 2014, 43, 5994.

173. Lustig, W. P.; Mukherjee, S.; Rudd, N. D.; Desai, A. V.; Li, J.; Ghosh, S. K.; Chem. Soc. Rev. 2017, 46, 3242.
174. Simon, C. M.; Braun, E.; Carraro, C.; Smit, B.; Proc. Natl. Acad. Sci. U. S. A. 2017, 114, 287.

175. Barea, E.; Montoro, C.; Navarro, J. A. R.; Chem. Soc. Rev. 2014, 43, 5419.

176. Denny Jr., M. S.; Moreton, J. C.; Benz, L.; Cohen, S. M.; Nat. Rev. Mat. 2016, 1, 16078.

177. Banerjee, D.; Cairns, A. J.; Liu, J.; Motkuri, R. K.; Nune, S. K.; Fernandez, C. A.; Krishna, R.; Strachan, D. M.; Thallapally, P. K.; Acc. Chem. Res. 2015, 48, 211.

178. Chughtai, A. H.; Ahmad, N.; Younus, H. A.; Laypkov, A.; Verpoort, F.; Chem. Soc. Rev. 2015, 44, 6804.

179. Alegre-Requena, J. V.; Marqués-López, E.; Herrera, R. P.; Díaz, D. D.; CrystEngComm 2016, 18, 3985.

180. Huang, Y.-B.; Liang, J.; Wang, X.-S.; Cao, R.; Chem. Soc. Rev. 2017, 46, 126.

181. Zhao, X.; Liu, S.; Tang, Z.; Niu, H.; Cai, Y.; Meng, W.; Wu, F.; Giesy, J. P.; Sci. Rep. 2015, 5, 11849.

182. Wang, L.; Han, Y.; Feng, X.; Zhou, J.; Qi, P.; Wang, B.; Coord. Chem. Rev. 2015, 307, 361.

183. Ullman, A. M.; Brown, J. W.; Foster, M. E.; Léonard, F.; Leong, K.; Stavila, V.; Allendorf, M. D.; Inorg. Chem. 2016, 55, 7233.

184. bp.com/statisticalreview, acessada em agosto de 2018.

185. http://data.worldbank.org/indicator/EN.ATM.CO2E.KT, acessada em agosto de 2018

186. Schoedel, A.; Ji, Z.; Yaghi, O. M.; Nat. Energy 2016, 1, 16034.

187. http://www.emercedesbenz.com/autos/mercedes-benz/concept-vehicles/ mercedes-benz-f125-research-vehicle-technology, acessada em agosto de 2018.

188. http://www.interacademycouncil.net/File.aspx $? i d=24548$, acessada em agosto de 2018

189. Langmi, H. W.; Ren, J.; North, B.; Mathe, M.; Bessarabov, D.; Electrochim. Acta 2014, 128, 368.

190. Furukawa, H.; Miller, M. A.; Yaghi, O. M.; J. Mater. Chem. 2007, 17, 3197.

191. Rowsell, J. L. C.; Yaghi, O. M.; Angew. Chem., Int. Ed. 2005, 44, 4670.

192. Dinča, M.; Dailly, A.; Liu, Y.; Brown, C. M.; Neumann, D. A.; Long, J. R.; J. Am. Chem. Soc. 2006, 128, 16876.

193. Yan, Y.; Blake, A. J.; Lewis, W.; Barnett, S. A.; Dailly, A.; Champness, N. R.; Schröder, M.; Chem. - Eur. J. 2011, 17, 11162.

194. Schlichtenmayer, M.; Hirscher, M.; J. Mater. Chem. 2012, 22, 10134.

195. Villajos, J. A.; Orcajo, G.; Martos, C.; Botas, J. Á.; Villacañas, J.; Calleja, G.; Int. J. Hydrogen Energy 2015, 40, 5346.

196. Hirscher, M.; Panella, B.; Schmitz, B.; Microporous Mesoporous Mater. 2010, 129, 335.

197. Falcaro, P.; Ricco, R.; Yazdi, A.; Imaz, I.; Furukawa, S.; Maspoch, D.; Ameloot, R.; Evans, J. D.; Doonan, C. J.; Coord. Chem. Rev. 2016, 307, 237.

198. Zlotea, C.; Campesi, R.; Cuevas, F.; Leroy, E.; Dibandjo, P.; Volkringer, C.; Loiseau, T.; Férey, G.; Latroche, M.; J. Am. Chem. Soc. 2010, 132, 2991.

199. Li, P.; Wang, Z.; Qiao, Z.; Liu, Y.; Cao, X.; Li, W.; Wang, J.; Wang, S.; J. Membr. Sci. 2015, 495, 130 .

200. Kuppler, R. J.; Timmons, D. J.; Fang, Q. R.; Li, J. R.; Makal, T. A.; Young, M. D.; Yuan, D.; Zhao, D.; Zhuang, W.; Zhou, H. C.; Coord. Chem. Rev. 2009, 253, 3042.

201. Jin, H.; Wollbrink, A.; Yao, R.; Li, Y.; Caro, J.; Yang, W.; J. Membr. Sci. 2016, 513, 40.

202.https://science.energy.gov/ /media/bes/pdf/reports/files/Basic_Research_ Needs_for_Carbon_Capture_rpt.pdf, acessada em agosto de 2018 .

203. https://climatechangeconnection.org/emissions/co2-equivalents/ , acessada em agosto de 2018

204. He, Y.; Zhou, W.; Qian, G.; Chen, B.; Chem. Soc. Rev. 2014, 43, 5657. 
205. Koh, H. S.; Rana, M. K.; Wong-Foy, A. G.; Siegel, D. J.; J. Phys. Chem. C 2015, 119, 13451.

206. Mason, J. A.; Oktawiec, J.; Taylor, M. K.; Hudson, M. R.; Rodriguez, J.; Bachman, J. E.; Gonzalez, M. I.; Cervellino, A.; Guagliardi, A.; Brown, C. M.; Llewellyn, P. L.; Masciocchi, N.; Long, J. R.; Nature 2015, 527, 357.

207. Peng, Y.; Krungleviciute, V.; Eryazici, I.; Hupp, J. T.; Farha, O. K.; Yildirim, T.; J. Am. Chem. Soc. 2013, 135, 11887.

208. Simon, C. M.; Kim, J.; Gomez-Gualdron, D. A.; Camp, J. S.; Chung, Y. G.; Martin, R. L.; Mercado, R.; Deem, M. W.; Gunter, D.; Haranczyk, M.; Sholl, D. S.; Snurr, R. Q.; Smit, B.; Energy Environ. Sci. 2015, 8, 1190.

209. Eddaoudi, M.; Kim, J.; Rosi, N.; Vodak, D.; Wachter, J.; O'Keeffe, M.; Yaghi, O. M.; Science 2002, 295, 469.

210. Gándara, F.; Furukawa, H.; Lee, S.; Yaghi, O. M.; J. Am. Chem. Soc. 2014, 136, 5271.

211. Guo, Z.; Wu, H.; Srinivas, G.; Zhou, Y.; Xiang, S.; Chen, Z.; Yang, Y.; Zhou, W.; O'Keeffe, M.; Chen, B.; Angew. Chem., Int. Ed. 2011, 50, 3178.

212. He, Y.; Zhou, W.; Yildirim, T.; Chen, B. Energy Environ. Sci. 2013, 6, 2735.

213. Wen, H. M.; Li, B.; Li, L.; Lin, R. B.; Zhou, W.; Qian, G.; Chen, B.; Adv. Mater. 2018, 30, 1704792.

214. Alezi, D.; Belmabkhout, Y.; Suyetin, M.; Bhatt, P. M.; Weseli ski, Ł. J.; Solovyeva, V.; Adil, K.; Spanopoulos, I.; Trikalitis, P. N.; Emwas, A. H.; Eddaoudi, M.; J. Am. Chem. Soc. 2015, 137, 13308.

215. http://www.ecofuel-asia-tour.com, acessada em agosto de 2018.

216. Li, B.; Wen, H.-M.; Wang, H.; Wu, H.; Yildirim, T.; Zhou, W.; Chen, B.; Energy Environ. Sci. 2015, 8, 2504.

217. Szilágyi, P. Á.; Serra-Crespo, P.; Gascon, J.; Geerlings, H.; Dam, B.; Front. Energy Res. 2016, 4, 2012.

218. Jiang, J.; Furukawa, H.; Zhang, Y. B.; Yaghi, O. M.; J. Am. Chem. Soc. 2016, 138, 10244.

219. Schneemann, A.; Bon, V.; Schwedler, I.; Senkovska, I.; Kaskel, S.; Fischer, R. A.; Chem. Soc. Rev. 2014, 43, 6062.

220. Zhou, K.; Chaemchuen, S.; Verpoort, F.; Renewable Sustainable Energy Rev. 2017, 79, 1414.

221. Pires, J.; Ju ków, J.; Pinto, M. L.; Colloids Surf., A 2018, 544, 105.

222. Yang, T.; Lu, L.; Wang, S.; Cao, W.; Lu, X.; Mol. Simul. 2017, 7022, 1.

223. Mafra, L.; endak, T.; Schneider, S.; Wiper, P. V.; Pires, J.; Gomes, J. R. B.; Pinto, M. L.; Chem. Eng. J. 2018, 336, 612.

224. Billemont, P.; Heymans, N.; Normand, P.; De Weireld, G.; Adsorption 2017, 23, 225.

225. Chen, C. X.; Zheng, S. P.; Wei, Z. W.; Cao, C. C.; Wang, H. P.; Wang, D.; Jiang, J. J.; Fenske, D.; Su, C. Y.; Chem. - Eur. J. 2017, 23, 4060.

226. Kong, C.; Du, H.; Chen, L.; Chen, B.; Energy Environ. Sci. 2017, 10, 1812.

227. Peng, Y.; Li, Y.; Ban, Y.; Yang, W.; Angew. Chem., Int. Ed. 2017, 56, 9757.

228. Sabetghadam, A.; Seoane, B.; Keskin, D.; Duim, N.; Rodenas, T.; Shahid, S.; Sorribas, S.; Guillouzer, C. Le; Clet, G.; Tellez, C.; Daturi, M.; Coronas, J.; Kapteijn, F.; Gascon, J.; Adv. Funct. Mater. 2016, 26 , 3154.

229. Ferreira, A. F. P.; Ribeiro, A. M.; Kulaç, S.; Rodrigues, A. E.; Chem. Eng. Sci. 2015, 124, 79.

230. Cheng, Y.; Wang, X.; Jia, C.; Wang, Y.; Zhai, L.; Wang, Q.; Zhao, D.; J. Membr. Sci. 2017, 539, 213.

231. Li, J. R.; Ma, Y.; McCarthy, M. C.; Sculley, J.; Yu, J.; Jeong, H. K.; Balbuena, P. B.; Zhou, H. C.; Coord. Chem. Rev. 2011, 255, 1791.
232. Millward, A. R.; Yaghi, O. M.; J. Am. Chem. Soc. 2005, 127, 17998.

233. Liu, J.; Thallapally, P. K.; McGrail, B. P.; Brown, D. R.; Liu, J.; Chem. Soc. Rev. 2012, 41, 2308.

234. Sabouni, R.; Kazemian, H.; Rohani, S.; Environ. Sci. Pollut. Res. 2014, $21,5427$.

235. Zhao, P.; Lampronti, G. I.; Lloyd, G. O.; Suard, E.; Redfern, S. A. T.; J. Mater. Chem. A 2014, 2, 620.

236. Chen, S.; Lucier, B. E. G.; Boyle, P. D.; Huang, Y.; Chem. Mater. 2016, $28,5829$.

237. Zhang, J.; Shreeve, J. M.; Dalton Trans. 2016, 45, 2363.

238. Caskey, S. R.; Wong-Foy, A. G.; Matzger, A. J.; J. Am. Chem. Soc. 2008, 130, 10870.

239. Li, B.; Zhang, Z.; Li, Y.; Yao, K.; Zhu, Y.; Deng, Z.; Yang, F.; Zhou, X.; Li, G.; Wu, H.; Nijem, N.; Chabal, Y. J.; Lai, Z.; Han, Y.; Shi, Z.; Feng, S.; Li, J.; Angew. Chem., Int. Ed. 2012, 51, 1412.

240. Nugent, P.; Belmabkhout, Y.; Burd, S. D.; Cairns, A. J.; Luebke, R.; Forrest, K.; Pham, T.; Ma, S.; Space, B.; Wojtas, L.; Eddaoudi, M.; Zaworotko, M. J.; Nature 2013, 495, 80.

241. Beyzavi, M. H.; Stephenson, C. J.; Liu, Y.; Karagiaridi, O.; Hupp, J. T.; Farha, O. K.; Front. Energy Res. 2015, 2, 1.

242. Deria, P.; Li, S.; Zhang, H.; Snurr, R. Q.; Hupp, J. T.; Farha, O. K.; Chem. Commun. 2015, 51, 12478.

243. Zhang, T.; Lin, W.; Chem. Soc. Rev. 2014, 43, 5982.

244. Wang, C. C.; Zhang, Y. Q.; Li, J.; Wang, P.; J. Mol. Struct. 2015, 1083, 127.

245. Roy, S. C.; Varghese, O. K.; Paulose, M.; Grimes, C. a.; ACS Nano 2010 , 4, 1259.

246. Corma, A.; Garcia, H.; J. Catal. 2013, 308, 168.

247. Ozin, G. A.; Adv. Mater. 2015, 27, 1957.

248. Dhakshinamoorthy, A.; Asiri, A. M.; García, H.; Angew. Chem., Int. Ed. 2016, 55, 5414.

249. Goeppert, A.; Czaun, M.; Jones, J.-P.; Prakash, G. K. S.; Olah, G. A.; Chem. Soc. Rev. 2014, 43, 7995.

250. Wang, D.; Huang, R.; Liu, W.; Sun, D.; Li, Z.; ACS Catal. 2014, 4, 4254.

251. Cardoso, J. C.; Stulp, S.; de Brito, J. F.; Flor, J. B. S.; Frem, R. C. G.; Zanoni, M. V. B.; Appl. Catal., B 2018, 225.

252. Lu, G.; Farha, O. K.; Zhang, W.; Huo, F.; Hupp, J. T.; Adv. Mater. 2012 , 24,3970

253. United Nations World Water Assessment Programme / UN-Water; The United Nations World Water Development Report 2018: Nature-Based Solutions for Water. Paris, UNESCO, 2018.

254. http://www.mma.gov.br/estruturas/sedr_proecotur/_publicacao/140_ publicacao09062009025910.pdf. Acessada em agosto de 2018.

255. United Nations World Water Assessment Programme; The United Nations World Water Development Report 4: Managing Water under Uncertainty and Risk. Paris, UNESCO, 2012, 1.

256. Shourideh, A. H.; Ajram, W. B.; Lami, J. A.; Haggag, S.; Mansouri, A.; Therm. Sci. Eng. Prog. 2018, 6, 14.

257. Furukawa, H.; Gándara, F.; Zhang, Y.-B.; Jiang, J.; Queen, W. L.; Hudson, M. R.; Yaghi, O. M.; J. Am. Chem. Soc. 2014, 136, 4369.

258. Rieth, A. J.; Yang, S.; Wang, E. N.; Dincă, M.; ACS Cent. Sci. 2017, 3, 668.

259. Kim, H.; Yang, S.; Rao, S. R.; Narayanan, S.; Kapustin, E. A.; Furukawa, H.; Umans, A. S.; Yaghi, O. M.; Wang, E. N.; Science 2017, 356, 430.

260. Kim, H.; Rao, S. R.; Kapustin, E. A.; Zhao, L.; Yang, S.; Yaghi, O. M.; Wang, E. N.; Nat. Commun. 2018, 9, 1191. 